Zbl Arbeitsmed 2017 67:64-77

DOI 10.1007/s40664-016-0150-4

Online publiziert: 26 . Oktober 2016

(c) Der/die Autor(en) 2016. Dieser Artikel ist eine Open-Access-Publikation.

CrossMark

\section{Brütting ${ }^{1} \cdot$ I. Hermanns ${ }^{1} \cdot$ A. Nienhaus ${ }^{2} \cdot$ R. Ellegast ${ }^{1}$}

'Institut für Arbeitsschutz der Deutschen Gesetzlichen Unfallversicherung (IFA), Sankt Augustin, Deutschland

${ }^{2}$ Berufsgenossenschaft für Gesundheitsdienst und Wohlfahrtspflege (BGW), Hamburg, Deutschland

\title{
Muskel-Skelett-Belastungen beim Schieben und Ziehen von Krankenbetten und Rollstühlen
}

Kranken- und Pflegepersonal ist aufgrund der körperlich belastenden Tätigkeiten hohen gesundheitlichen Beanspruchungen ausgesetzt. Bei der stationären Krankenpflege müssen beispielsweise Patienten und Pflegebedürftige angehoben, umgelagert, mobilisiert, gestützt und transportiert werden. Laut dem Bundesministerium für Gesundheit und dem BKK Bundesverband [5] wird sich die Zahl der Pflegebedürftigen bis 2050 auf etwa 4,4 Mio. etwa verdoppeln. Allein 500.000 Pflegefachkräfte müssten in den kommenden 20 Jahren zusätzlich qualifiziert werden. „In der Altenpflege wird bis zum Jahr 2050 eine Zunahme der Altenpflegekräfte um lediglich $30 \%$ prognostiziert - während aber die Anzahl der Pflegefälle um voraussichtlich etwa $270 \%$ steigen soll. Eine vom Forschungsinstitut Betriebliche Bildung (f-bb) durchgeführte bundesweite Befragung von Pflegedienstleitungen in Krankenhäusern [16] unterstreicht die Brisanzder Alterung von Beschäftigten in der Pflege: Mehr als die Hälfte (56 \%) der befragten Pflegedienstleister bestätigen schon heute ein hohes Durchschnittsalter bei den Belegschaften. Hierbei rechnen $59 \%$ mit zunehmenden Leistungseinschränkungen und Fehltagen angesichts des prognostizierten Anstiegs älterer Pflegekräfte. Insgesamt $29 \%$ der Krankheitstage bei Krankenschwestern sind auf Muskel-Skelett-Erkrankungen (MSE) zurückzuführen [11]. Immer mehr ältere Patienten und Pflegebedürftige werden von weniger und älter werdenden Fachkräften gepflegt werden müssen." [5]. Mit der Aufhebung der Wehrpflicht 2011 ging ein weiterer Verlust von Pflegekräften (Zivildienstleistende) einher. Bezogen auf in Krankenhäusern und Kliniken Beschäftigte entfallen laut dem Fehlzeitenreport von 2004 [1] 608 Krankheitstage je 100 bei der AOK Versicherte bei einer Krankheitsdauer von durchschnittlich 19,2 Tagen auf MSE (Branchendurchschnitt: 556 Krankheitstage bzw. 11,8 Tage Krankheitsdauer). Um den Pflegeberuf zukünftig attraktiv zu gestalten, müssen Arbeitsabläufe ergonomisch und belastungsoptimiert gestaltet sein. Dabei ist als Ausgangspunkt eine detailliertere Analyse der körperlich belastenden Tätigkeiten in Pflegeberufen notwendig.
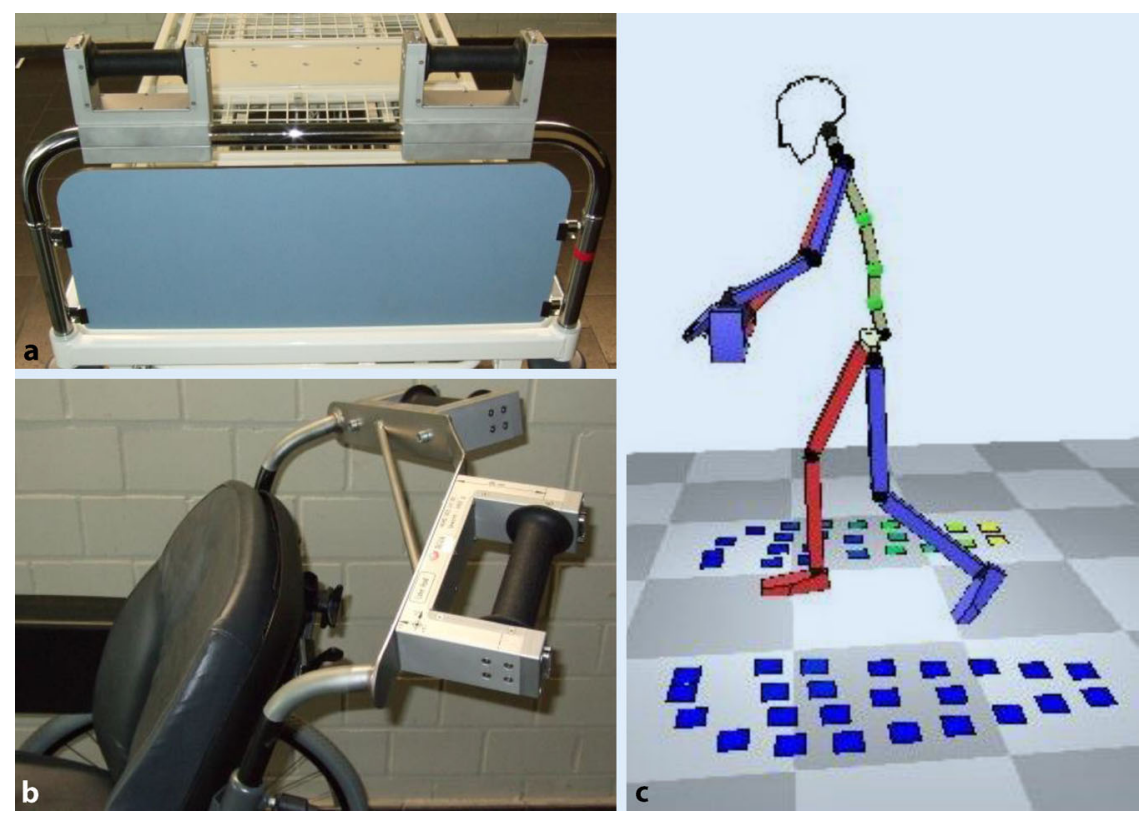

Abb. 1a,b $\Delta$ Krankenbett und Rollstuhl mit Kraftgriffen zur Handkraftmessung. c CUELA-Messwertdarstellung 


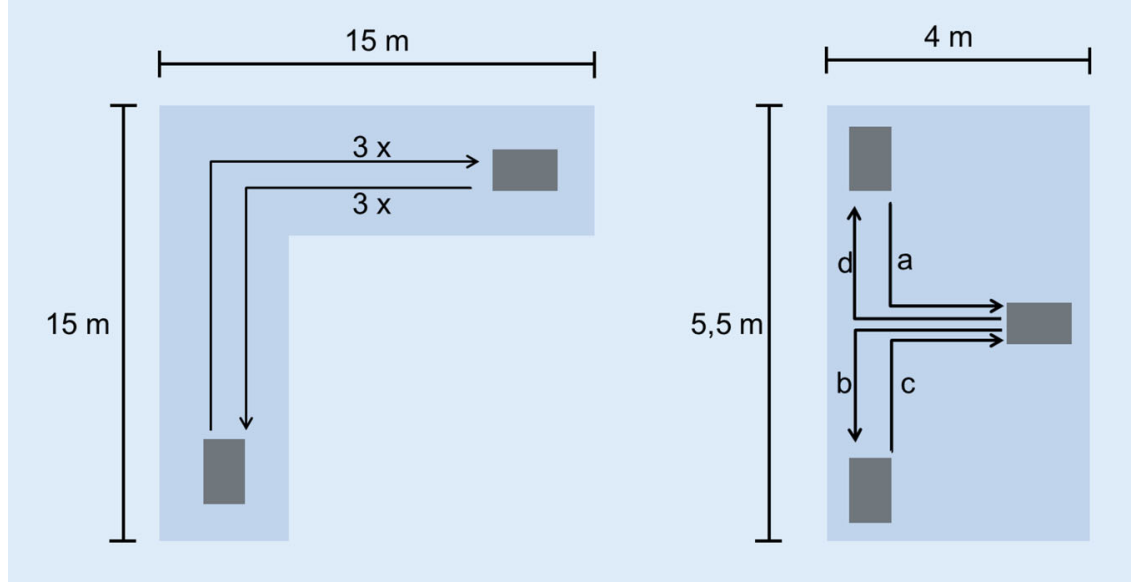

Abb. $2 \triangle$ Ziehen und Schieben über einen langen Flur und Manövrieren in einem Zimmer

kenbetten und Rollstühlen durchgeführt. Ziel war es, äußere Belastungsfaktoren des Muskel-Skelett-Systems zu quantifizieren und tätigkeitsspezifische Lumbalbelastungen (Kompressionskräfte und Drehmomente im Lendenwirbelsäulenbereich L5/S1) durch biomechanische Modellrechnungen abzuschätzen.

\section{Methodik}

\section{Material}

Für die Schiebe- und Ziehversuche wurden ein typisches Krankenbett (Hersteller: Joh. Stiegelmeyer \& Co. GmbH, Herford, Modell Vivendo) und ein Krankenrollstuhl (Hersteller: Handicare GmbH, Minden, Modell Cirrus) verwendet. Die Versuche wurden überwiegend auf gefliestem Boden durchgeführt; einige Messungen mit dem Rollstuhl fanden teilweise auf Teppichboden (Rampe) statt. Experten der Berufsgenossenschaft für Gesundheitsdienst und Wohlfahrtspflege (BGW) schätzten die Rollreibungseigenschaften der Laborböden zuvor als vergleichbar mit denen in Krankenhäusern und Pflegeeinrichtungen ein.

\section{Probanden}

Die Versuche wurden von 10 Probanden ( 5 Männer und 5 Frauen, Alter: 22 bis 43 Jahre, Frauen: 24,2 $\pm 2,2$ Jahre, Männer: 32,4 $\pm 7,0$ Jahre), bei denen es sich um Mitarbeiter des IFA und keine ausgebildeten Pflegekräfte handelte, je 3-mal durchgeführt. Der Mittelwert der Kör- perhöhe betrug bei den Frauen 170,4 \pm $3,7 \mathrm{~cm}$, bei den Männern 185,2 $\pm 9,3 \mathrm{~cm}$ bei einem mittleren Körpergewicht von $65,4 \pm 3,2 \mathrm{~kg}$ der Frauen und 90,8 \pm $16,9 \mathrm{~kg}$ der Männer.

\section{Messtechnik}

Handkräfte beim Ziehen und Schieben wurden mit einem im IFA entwickelten 3D-Handkraftmesssystem (HKMS, [17]) gemessen, welches am o.g. Krankenbett und Rollstuhl angebracht wurde (- Abb. 1). Dabei wurde darauf geachtet, dass die Kraftmessgriffe der ursprünglichen Position und Lage der Anfasspunkte am Rollstuhl und Bett entsprechen. Die Höhe der Kraftgriffe wurde durch die Verstellmechanismen an Bett und Rollstuhl jeweils entsprechend der Körperhöhe der Probanden variiert. Die individuelle Griffhöhe war somit für die einzelnen Probanden bei allen Versuchsteilen gleich und entsprach der Mitte zwischen Handgelenk und Ellenbogen bei aufrechtem Stand und nach unten ausgestreckten Armen. Somit wurde eine etwa annähernd horizontale Krafteinwirkung, die nach Looze et al. [4] ein effizientes Schieben und Ziehen ermöglicht, eingehalten. Identische Griffhöhen hätten zur Folge gehabt, dass unterschiedliche Körperhöhen der Probanden zu unterschiedlichen Winkeln der Krafteinleitung geführt hätten. Als Resultat würde z. B. bei größeren Probanden die Berechnung der Kompressionskraft auf L5/S1 beim Schieben proportional kleinere Werte liefern als bei kleineren Probanden, bei denen die Kraftrichtung in diesem Fall weniger nach unten gerichtet gewesen wäre. Der horizontale Abstand der beiden Kraftgriffe voneinander blieb konstant und betrug bauartbedingt beim Rollstuhl $40 \mathrm{~cm}$ und beim Bett $50 \mathrm{~cm}$.

\section{》) In dieser Studie wurden ins- besondere die Neigungswinkel an Brust- und Lendenwirbelsäule betrachtet}

Weiterhin wurde die Körperhaltung der Probanden während der Versuche mit einem Körperhaltungsmesssystem aufgezeichnet. Das hierfür verwendete CUELA-System $[9,10]$ erfasst mit einer Frequenz von $50 \mathrm{~Hz}$ die Gelenkwinkel der Beine, des Rumpfes und der Arme sowie die Daten der Kraftgriffe. Mit diesen Daten lassen sich die Körperhaltung, der zeitliche Verlauf der Bewegungen und die Aktionskräfte dreidimensional darstellen. In dieser Studie wurden insbesondere die Neigungswinkel an Brust(BWS) und Lendenwirbelsäule (LWS) betrachtet. Aus diesen beiden Neigungswinkeln wurden mittlere Rumpfneigungen (= [LWS-Winkel + BWS-Winkel]/2) und Rückenkrümmungen (= BWS-Winkel - LWS-Winkel) berechnet und deskriptiv ausgewertet.

\section{Versuchsdesign: Bett}

Die typischen Fahrwege im Krankenhausbetrieb sind der Transport zwischen verschiedenen Räumen (z. B. zwischen Krankenzimmer und Operationssaal), und sie beinhalten das Schieben und/ oder Ziehen über Flure sowie das Manövrieren in einem Raum oder um Hindernisse herum (z. B. Sitzmöbel oder kurzzeitig abgestellte technische Geräte im Flur, Flurabzweigung o. Ä.). Für die experimentellen Versuche wurde deshalb die Fahrt über eine längere, gerade Strecke mit einer Kurve (Schieben bzw. Ziehen, Gesamtstrecke ca.30 m;• Abb. 2) simuliert. Das Manövrieren (Kombination aus Schieben und Ziehen) erfolgte innerhalb eines Rechtecks von etwa $4 \times$ $5,5 \mathrm{~m}$ und stellte die Situation in einem Krankenzimmer nach. 
Zbl Arbeitsmed 2017 · 67:64-77 DOI 10.1007/s40664-016-0150-4

(c) Der/die Autor(en) 2016. Dieser Artikel ist eine Open-Access-Publikation.

\section{Brütting $\cdot$ I. Hermanns $\cdot$ A. Nienhaus $\cdot$ R. Ellegast}

\section{Muskel-Skelett-Belastungen beim Schieben und Ziehen von Krankenbetten und Rollstühlen}

\section{Zusammenfassung}

Hintergrund. In dieser Pilotstudie wurden standardisierte Labormessungen physischer Belastungen beim Ziehen, Schieben und Manövrieren von Krankenbetten und Rollstühlen durchgeführt. Ziel war es, äußere Belastungsfaktoren des Muskel-Skelett-Systems zu quantifizieren und tätigkeitsbezogene Lumbalbelastungen abzuschätzen.

Methodik. Zehn Probanden führten standardisierte Schiebe- und Ziehtätigkeiten im Labor mit einem typischen Krankenbett und einem Krankenrollstuhl durch. Handkräfte wurden mit einem 3D-Handkraftmesssystem gemessen. Mit dem CUELA-Messsystem wurde der zeitliche Verlauf von Änderungen der Körperhaltungen, Gelenkwinkel der oberen und unteren Extremitäten und Rumpf/Rücken aufgezeichnet. Resultierende Drehmomente und Kompressionskräfte im Lendenwirbelsäulenbereich ( $L 5 / S 1)$ wurden mit einfachen biomechanischen Modellrechnungen abgeschätzt. Ergebnisse. Das Schieben, Ziehen und Manövrieren von Krankenbetten und Rollstühlen mit einem Gesamtgewicht von 240 bzw. $135 \mathrm{~kg}$ auf einer geraden Strecke erforderte im Mittel Handkräfte (Gesamtkraftvektor) von bis zu $79 \pm 7$ N (max. 368 N). Die Handkraftmessung beim Überwinden einer Stufe mit einem Rollstuhl (Dummygewicht: $100 \mathrm{~kg}$ ) ergab einen Maximalwert von 856 $\mathrm{N}$ (Median: $128 \pm 86 \mathrm{~N}$ und $221 \pm 184 \mathrm{~N}$ ). Das Schieben und Ziehen des Bettes und des Rollstuhls verursachte im 95. Perzentil Werte von bis zu $45 \mathrm{Nm}$ Drehmoment an und 1,2 kN Kompressionskraft auf L5/S1. Beim Manövrieren wurden teilweise lumbale Momente von über $80 \mathrm{Nm}$ und Kompressionskräfte über $1,6 \mathrm{kN}$ berechnet. Die Drehmomente und Kompressionskräfte beim
Schieben und Ziehen des Bettes erreichten während der Startphase höhere Werte als in den übrigen Phasen, was auf ungünstigere Körperhaltungen zurückgeführt werden kann. Diskussion. In dieser Untersuchung konnten äußere Muskel-Skelett-Belastungen und Lumbalbelastungen beim Ziehen und Schieben von Krankenbetten und Rollstühlen quantifiziert werden. Die Ergebnisse früherer Studien wurden mit Einschränkungen bestätigt. Zur Verifizierung der Ergebnisse sollten weitere Untersuchungen mit einem größeren Probandenkollektiv und unterschiedlichen Bodenbelägen durchgeführt werden.

Schlüsselwörter

Krankenbett · Rollstuhl · Handkraft · Körperhaltung · Bandscheibendruckkraft

\section{Musculoskeletal stress during pushing and pulling hospital beds and wheelchairs}

\section{Abstract}

Background. In this pilot study the physical stresses associated with pushing, pulling and maneuvering of hospital beds and wheelchairs were measured and recorded under standardized laboratory conditions. This enabled initial observations of the external stress on the musculoskeletal system to be quantified and the lumbar stress to be estimated.

Methods. A typical hospital bed and a hospital wheelchair were used by 10 subjects for standardized pushing and pulling tests. Hand forces were measured by a 3D measurement system in handles fitted to the hospital bed and wheelchair. The CUELA measurement system was used to record body posture, joint angles of the upper and lower extremities and of the torso/back. The resulting torques and compression forces in the lumbar spine region (L5/S1) were estimated using simple biomechanical model calculations.

Results. The pushing, pulling and maneuvering of hospital beds and wheelchairs with a total weight of 240 and $135 \mathrm{~kg}$ during straight line travel required mean hand forces of up to $79 \mathrm{~N} \pm 7 \mathrm{~N}$ (maximum value 368 $\mathrm{N})$. Measurement of the hand force during negotiation of a step with a wheelchair and a dummy weight of $100 \mathrm{~kg}$ yielded a maximum value of $856 \mathrm{~N}$ (mean $128 \mathrm{~N} \pm 86 \mathrm{~N}$ and $221 \mathrm{~N}$ $\pm 184 \mathrm{~N}$, respectively). Pushing and pulling of the bed and wheelchair resulted in torque values of up to $45 \mathrm{Nm}$ and compression forces of up to $1.2 \mathrm{kN}$ at the 95 th percentile. Torques of over $80 \mathrm{Nm}$ and compression forces of over $1.6 \mathrm{kN}$ were measured in some cases during maneuvering. The torques and compression forces generated at the L5/S1 level during pushing and pulling of the bed were higher at the beginning of pulling than in other phases. This can be attributed to unfavorable posture. Discussion. In this study forces and stresses arising during the pulling and pushing of hospital beds and wheelchairs were measured. The results of earlier studies were confirmed, subject to the constraints deriving from differences between the methods. The results should be verified by further studies employing a larger collective of test subjects and different floor coverings.

\section{Keywords}

Hospital bed - Wheelchair - Handforce . Posture - Disc compression force
Ein Versuchsdurchgang „Flur" bestand aus jeweils einem Hin- und Rückweg, ein Versuchsdurchgang „Manövrieren im Zimmer" aus Hin- und Rückweg mit jeweils einer Halteposition, vorwärts schiebend und rückwärts ziehend (• Abb. 2).

Das Ziehen des Bettes erfolgte rückwärtsgehend. Die Rollen bzw. Räder standen zu Versuchsbeginn in Fahrtrich- tung. Die Lenksperren der kopfseitigen Laufrollen des Bettes wurden für den Geradeauslauf eingerastet und für die Manövriervorgänge frei beweglich eingestellt. Das Leergewicht des Bettes (ohne Auflagen wie Matratze, Bettgarnitur usw.) betrug $140 \mathrm{~kg}$, die Kraftgriffe wogen inkl. Befestigung 5,6 kg. Unterschiedliche Patientengewichte wurden durch verschieden schwere Dummys (80 und $100 \mathrm{~kg}$ ) realisiert. Für die Ausführung der Zieh- und Schiebeaufgaben wurden keine Zeitlimits vorgegeben, die Ganggeschwindigkeit konnte somit frei gewählt werden. Zwischen den Wiederholungen standen den Probanden kurze Pausen zur Verfügung, während denen die Startbedingungen (Positionierung des Bettes bzw. des Rollstuhls und Aus- 


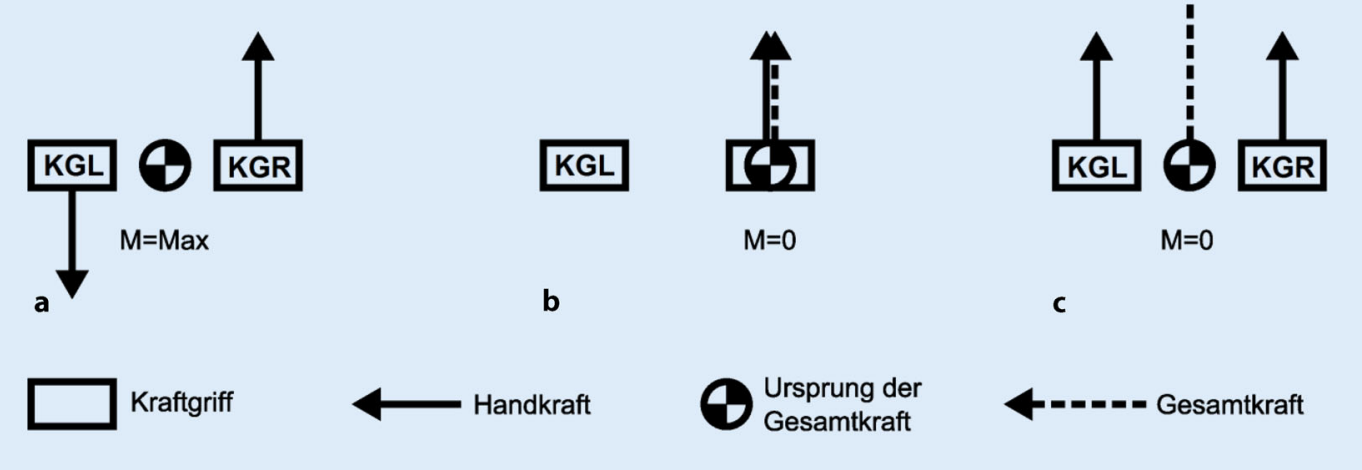

Abb. $3<$ Verschiedene Kraftfälle in der Draufsicht, resultierende Momente $(M)$ und vektorielle Gesamtkräfte. Die Pfeile der Handkräfte entsprechen den Kraftvektoren $\left(\overrightarrow{K G R_{F}}\right)$ und $\left(\overrightarrow{K G L_{F}}\right)$, die Positionen der Kraftgriffe entsprechen $\overrightarrow{K G L_{P o s}}$ und $\overrightarrow{K G R_{\text {Pos }}}$. Der Ursprung der Gesamtkraft entspricht $\overrightarrow{K G G e \text { Pos }_{\text {Po }}}$ richtung der Rollen) wiederhergestellt wurden.

\section{Versuchsdesign: Rollstuhl}

Auf die Kombinationen „Rollstuhl ziehen auflangem Flur“ und „Rollstuhl leer“ wurde verzichtet, da diese im täglichen Pflegebetrieb kaum vorkommen bzw. keine relevante körperliche Belastung darstellen. Ansonsten wurde derselbe Parcours wie mit dem Bett verwendet. In einem Zusatzversuch wurde mit einem Probanden exemplarisch das Schieben über eine Rampe (Neigungswinkel: $3,5^{\circ}$ ) sowie die Überwindung einer Stufe (Höhe: $13 \mathrm{~cm}$ ) mit dem Rollstuhl und dem $100 \mathrm{~kg}$ schweren Dummy durchgeführt.

Das Leergewicht des Rollstuhls betrug $35 \mathrm{~kg}$, die Kraftgriffe wogen inkl. Befestigung 5,6 kg. Unterschiedliche Patientengewichte wurden auch hier mit verschieden schweren Dummys (80 und $100 \mathrm{~kg}$ ) simuliert.

Aufgrund des anzunehmenden variierenden Kraftverlaufs innerhalb der Versuchsteile wurde beim Ziehen und Schieben auf gerader, längerer Strecke (Flur) eine Unterteilung in verschiedene Phasen vorgenommen, die als Startphase, Rollphase und Bremsphase bezeichnet werden und folgendermaßen definiert sind:

- Die Startphase („Start“) beginnt mit dem Start des Versuchs und endet nach dem 4. Schritt des Probanden, der durch eine Mustererkennung des Kniewinkelverlaufs mit dem CUELA-System erfasst wurde.

- Die Rollphase („Roll“) beginnt mit dem Abschluss des 4. Schritts und endet vor Beginn der letzten 4 Schritte. Eine Unterteilung der Rollphase in Geradeausfahrt und Kurvenfahrt wurde anhand der einsetzenden und endenden Lenkbewegungen vorgenommen.

- Die Bremsphase („Brems“) beinhaltet den Zeitraum der letzten 4 Schritte bis zum Stillstand des Bettes bzw. Rollstuhls.

Das Manövrieren stellt eine Ansammlung mehrerer aufeinanderfolgender Start-, Roll- und Bremsphasen mit gleichzeitigen Lenkbewegungen dar und wurde nicht in einzelne Phasen untergliedert.

\section{Auswertungsparameter}

Als Grundlage für die Abschätzung der Muskel-Skelett-Belastungen dienen in dieser Studie die im Folgenden aufgeführten Parameter.

\section{Gesamtkraft an Kraftgriffen (N)}

Die Gesamtkraft beider Hände ist definiert als die vektorielle Summe der jeweils 3 Komponenten beider Handkräfte (X-, Y- und Z-Richtung). Es werden folgende statistische Werte berechnet: Mittelwert (MW), Standardabweichung (SD), Maximum (Max) sowie die Perzentilwerte $5 \%$ (P05), $25 \%$ (P25), 50 \% (Median, P50), $75 \%$ (P75) und $95 \%$ (P95). Eine Bewertung der Kräfte erfolgt nach ISO 11228-2 [23] und DIN 33411-5 [6].

\section{Moment an Kraftgriffen (Nm)}

Das resultierende Moment zwischen beiden Kraftgriffen (KGR, KGL) dient als Maß für die Differenz hinsichtlich Kraftrichtung und -höhe (bei asymmetrischen Kraftaufwendungen beider Hände) und wird bei der Berechnung der Lumbalmomente und Kompressionskräfte an L5/S1 berücksichtigt. - Abb. 3 zeigt beispielhaft verschiedene vorkommende Kraftfälle für die Berechnung des resultierenden Handkraftmoments. Es wird in 2 Schritten berechnet. Im ersten Schritt erfolgt die Berechnung der Position eines virtuellen Gesamtkraftgriffs $\left(\overrightarrow{K_{G G e s_{P o s}}}\right)$ aus den Positionen der Kraftgriffe $\left(\overrightarrow{K G L_{P o s}}, \overrightarrow{K G R_{P o s}}\right)$ und den gemessenen Kräften beider Kraftgriffe $\left(\overrightarrow{K G R_{F}}, \overrightarrow{K G L_{F}}\right)$ :

$$
\begin{aligned}
& \overrightarrow{K G G e s_{P o s}}= \\
& \overrightarrow{K G L_{P o s}}+\left(\overrightarrow{K G R_{P o s}}-\overrightarrow{K G L_{P o s}}\right) \\
& * \frac{\left|\overrightarrow{K G R_{F}}\right|}{\left|\overrightarrow{K G R_{F}}\right|+\left|\overrightarrow{K G L_{F}}\right|} \\
& *\left(\overrightarrow{K G R_{F}}+\overrightarrow{K G L_{F}}\right) .
\end{aligned}
$$

Im zweiten Schritt wird das Moment aus der Position des Gesamtkraftgriffs $\left(\overrightarrow{\text { KGGes }_{M}}\right)$ und dem Kreuzprodukt der beiden Hebelarme der Kraftgriffe und deren Kräfte berechnet:

$$
\begin{aligned}
& \overrightarrow{\text { KGGes }_{M}}= \\
& \left(\overrightarrow{K G R_{\text {Pos }}}-\overrightarrow{K G G e s_{\text {Pos }}}\right) \times \overrightarrow{K G R_{F}} \\
& +\left(\overrightarrow{K G L_{\text {Pos }}}-\overrightarrow{\text { KGGes }}\right) \times \overrightarrow{K G L_{F}}
\end{aligned}
$$

a) Bei entgegengesetzten Kraftrichtungen an den Kraftgriffen entsteht ein maximales Moment um den Ursprung, da beide Kräfte mit dem 
Tab. 1 Schema zur Klassifikation von Momenten nach Tichauer [35, 36] und Kriterien nach Hecktor und Jäger [19]

\begin{tabular}{|c|c|c|c|c|}
\hline $\begin{array}{l}\text { Moment an } \\
\text { L5/S1 (ML5/S1) } \\
\text { in } \mathrm{Nm}\end{array}$ & $\begin{array}{l}\text { Belastung, } \\
\text { Arbeits- } \\
\text { schwere }\end{array}$ & $\begin{array}{l}\text { Anforderungen oder } \\
\text { Einschränkungen }\end{array}$ & $\begin{array}{l}\text { Belastungs- } \\
\text { stufe }\end{array}$ & $\begin{array}{l}\text { Belastungs- } \\
\text { intensität }\end{array}$ \\
\hline $\mathrm{ML} 5 / \mathrm{S} 1<40$ & $\begin{array}{l}\text { Leicht, } \\
\text { mühelos }\end{array}$ & $\begin{array}{l}\text { Ungeübte Personen, } \\
\text { Frauen oder Männer, } \\
\text { Konstitution unerheblich }\end{array}$ & 1 & $\begin{array}{l}\text { Gering } \\
\text { belastend }\end{array}$ \\
\hline $40 \leq \mathrm{ML} 5 / \mathrm{S} 1<85$ & Mittelschwer & $\begin{array}{l}\text { Guter Körperbau, einige } \\
\text { Übung }\end{array}$ & 2 & $\begin{array}{l}\text { Überlastung } \\
\text { möglich }\end{array}$ \\
\hline $\begin{array}{l}85 \leq \mathrm{ML} 5 / \mathrm{S} 1< \\
135\end{array}$ & Schwer & $\begin{array}{l}\text { Ausgewählte Personen, } \\
\text { eingehende Schulung, } \\
\text { Ruhepausen }\end{array}$ & 3 & $\begin{array}{l}\text { Überlastung } \\
\text { wahrscheinlich }\end{array}$ \\
\hline $135 \leq \mathrm{ML} 5 / \mathrm{S} 1$ & Sehr schwer & $\begin{array}{l}\text { Große Sorgfalt bei der } \\
\text { Personenauswahl und } \\
\text { Schulung, Schichtab- } \\
\text { schnitte }\end{array}$ & 4 & $\begin{array}{l}\text { Überlastung } \\
\text { sehr wahr- } \\
\text { scheinlich }\end{array}$ \\
\hline
\end{tabular}

Tab. 2 Mittlerer Zeitaufwand $(\mathrm{min})$ für Schiebe- und Ziehvorgänge (je 3 Hin- und Rückwege $\hat{=}$ $180 \mathrm{~m}$ ) und mittlere Ganggeschwindigkeit $\left(\mathrm{ms}^{-1}\right)$ für Frauen (w) und Männer ( $\mathrm{m}$ )

Ziehen $(\mathrm{w} / \mathrm{m})$

$\begin{array}{lll}\text { Leer } & 80 \mathrm{~kg} & 100 \mathrm{~kg} \\ \begin{array}{l}4,05 / 3,86 \mathrm{~min}\left(0,74 / 0,77 \mathrm{~ms}^{-1}\right) \\ \text { Schieben }(\mathrm{w} / \mathrm{m})\end{array} & 4,02 / 4,08 \mathrm{~min}\left(0,75 / 0,74 \mathrm{~ms}^{-1}\right) & 3,86 / 4,15 \mathrm{~min}\left(0,78 / 0,72 \mathrm{~ms}^{-1}\right) \\ \text { Leer } & 80 \mathrm{~kg} & 100 \mathrm{~kg} \\ 3,16 / 3,25 \mathrm{~min}\left(0,95 / 0,92 \mathrm{~ms}^{-1}\right) & 3,46 / 3,61 \mathrm{~min}\left(0,87 / 0,83 \mathrm{~ms}^{-1}\right) & 3,35 / 3,68 \mathrm{~min}\left(0,90 / 0,82 \mathrm{~ms}^{-1}\right)\end{array}$

maximalen Hebelarm in die Berechnung eingehen. Die vektorielle Gesamtkraft kann in diesem Fall auch 0 sein.

b) Bei Kraftausübung an nur einem Kraftgriff entsteht kein Moment, da der Ursprung der Gesamtkraft gleich dem Ursprung der einzelnen Kraft ist und somit kein Hebelarm existiert. Die vektorielle Gesamtkraft entspricht der Kraft an dem einzelnen Kraftgriff.

c) Bei gleichgerichteter Kraftausübung an beiden Kraftgriffen ist der Ursprung der Gesamtkraft mittig zwischen beiden Kraftgriffen und jede Kraft geht mit ihrem Hebelarm in das Moment ein. Das Moment kann 0 sein, wenn die Kräfte in beiden Kraftgriffen gleich hoch und gleich gerichtet sind. Die Gesamtkraft entspricht der vektoriellen Summe beider Kraftgriffe.

Ein resultierendes Moment von $50 \mathrm{Nm}$ zwischen den $50 \mathrm{~cm}$ voneinander entfernten Kraftgriffen kann z. B. durch das Ziehen in waagerechter Richtung mit $100 \mathrm{~N}$ an einem Kraftgriff und gleich- zeitiges Schieben am anderen Kraftgriff mit $100 \mathrm{~N}$ entstehen.

\section{Moment an L5/S1 (Nm)}

Die Körperhaltungen und Gelenkwinkel sind wesentlicher Bestandteil der Berechnung des Moments an und der Kompressionskraft auf L5/S1. Das zur Messung und Aufzeichnung dieser Parameter verwendete CUELA-System liefert mit seinen unterschiedlichen am Körper angebrachten Sensoren Winkelwerte für die Kopfhaltung, Armhaltung, Rumpfhaltung und Beinhaltung. Aus den Körperhaltungs- und Kraftgriffdaten wurden mittels dreidimensionaler biomechanischer Modellrechnungen mit 8 Segmenten (je 3 Armsegmente, Kopf und Rumpf mit jeweils definierten Massen, Maßen und Segmentschwerpunkten) oberhalb von L5/S1 das Moment an der Bandscheibe L5/S1 $\left(\overrightarrow{M_{L 5 S 1}}\right)$ berechnet (in Anlehnung an Glitsch et al. [18]). Dieses Moment besteht zum einen aus dem Körpermoment (Summe aller Segmentgewichte multipliziert mit dem jeweiligen Hebelarm des Segmentschwerpunkts von L5/S1) und zum anderen aus dem Moment resultierend aus den Kraftgriffen (Gesamtkraft der Kraftgriffe multipliziert mit dem Hebelarm). Das Moment wird quasistatisch berechnet, d. h. dynamische Effekte aufgrund der Massenträgheit werden als vernachlässigbar klein angenommen:

$$
\begin{aligned}
& \overrightarrow{M_{L 5 S 1}}= \\
& \sum_{l=1}^{8}\left(\overrightarrow{L_{5 S 1}}-\overrightarrow{S_{e g}}\right) \times\left(m_{S e g_{l}} * \vec{g}\right) \\
& +\overrightarrow{\text { KGGes }_{M}}+\left(\overrightarrow{L_{S S 1}}-\overrightarrow{\text { KGGes }_{\text {Pos }}}\right) \\
& \times \overrightarrow{\text { KGGes }_{\mathrm{F}}}
\end{aligned}
$$

Der Betrag des lumbalen Gesamtmomentenvektors wurde nach dem von Tichauer [36] definierten Schema zur Klassifikation von Momenten an L5/S1 bewertet (•Tab. 1):

\section{Kompressionskraft auf L5/S1 (kN)}

Basierend auf dem Moment an L5/S1 wird anhand eines sehr einfachen Muskelmodells die Kompressionskraft abgeschätzt. Das Muskelmodell enthält zur eindeutigen Lösung der Momentengleichung für die zu kompensierenden Momente einen Rückenstreckermuskel mit fest definierter anatomischer Geometrie [9]. Hiermit erfolgte die Abschätzung der Kompressionskraft auf L5/S1 unter Vernachlässigung der Seitbeuge- und Torsionsmomente - verursacht durch asymmetrische Körperhaltungen und/ oder Kraftausübungen. Eine Bewertung der Kräfte erfolgt nach den „Dortmunder Richtwerten“ [24].

\section{Statistische Auswertung}

Die Auswertung der Versuchsdaten erfolgte mit den folgenden statistischen Verfahren und Programmen: Die Darstellung von Verteilungen der Mittelwerte erfolgt in Boxplots. Die Box entspricht hierbei dem Wertebereich, in dem die mittleren $50 \%$ der Daten liegen. Sie wird durch das 25. und 75. Perzentil der Daten begrenzt. Der Median (50. Perzentil) ist als durchgehender Querstrich in der Box eingezeichnet. Die Antennen (Whiskers) markieren jeweils das 5. und 95. Perzentil der Werteverteilung. Die Boxplots wurden mit der im IFA entwickelten Auswertesoftware 
Tab. 3 Gesamtkräfte ( $\mathrm{N}$ ) für das Manövrieren $(M)$, Schieben $(S)$ und Ziehen $(Z)$ des Bettes mit

unterschiedlichen Dummygewichten (leer, 80 und $100 \mathrm{~kg}$ ). Werte sind zeitgewichtete Mittelwerte

der Einzelversuche; Ausnahme Max (absoluter maximaler Einzelwert)

\begin{tabular}{|c|c|c|c|c|c|c|c|c|c|}
\hline $\begin{array}{l}\text { Gewicht } \\
\text { Richtung }\end{array}$ & $\begin{array}{l}\text { Leer } \\
M\end{array}$ & $\begin{array}{l}\text { Leer } \\
S\end{array}$ & $\begin{array}{l}\text { Leer } \\
Z\end{array}$ & $\begin{array}{l}80 \mathrm{~kg} \\
M\end{array}$ & $\begin{array}{l}80 \mathrm{~kg} \\
S\end{array}$ & $\begin{array}{l}80 \mathrm{~kg} \\
Z\end{array}$ & $\begin{array}{l}100 \mathrm{~kg} \\
M\end{array}$ & $\begin{array}{l}100 \mathrm{~kg} \\
\mathrm{~S}\end{array}$ & $\begin{array}{l}100 \mathrm{~kg} \\
Z\end{array}$ \\
\hline P05 & 20 & 32 & 25 & 23 & 34 & 26 & 23 & 37 & 28 \\
\hline P25 & 39 & 49 & 36 & 48 & 53 & 39 & 48 & 59 & 41 \\
\hline P50 & 54 & 61 & 44 & 69 & 68 & 49 & 71 & 74 & 52 \\
\hline P75 & 76 & 77 & 54 & 95 & 86 & 62 & 103 & 93 & 65 \\
\hline P95 & 122 & 106 & 72 & 144 & 119 & 84 & 161 & 124 & 86 \\
\hline MW & 60 & 64 & 46 & 74 & 71 & 51 & 79 & 77 & 54 \\
\hline SD & 7 & 17 & 15 & 8 & 19 & 18 & 7 & 20 & 19 \\
\hline Max & 272 & 301 & 273 & 330 & 326 & 368 & 327 & 334 & 327 \\
\hline
\end{tabular}

Tab. 4 Gesamtkräfte (N) für das Manövrieren $(M)$ und Schieben (S) des Rollstuhls mit Dummygewichten von 80 und $100 \mathrm{~kg}$. Werte sind zeitgewichtete Mittelwerte der Einzelversuche; Ausnahme Max (absoluter maximaler Einzelwert)

\begin{tabular}{lllll}
\hline Gewicht & $\mathbf{8 0} \mathbf{~ k g}$ & $\mathbf{8 0} \mathbf{k g}$ & $\mathbf{1 0 0} \mathbf{~ k g}$ & $\mathbf{1 0 0} \mathbf{~ g g}$ \\
\hline Richtung & $\mathbf{M}$ & $\mathbf{S}$ & $\mathbf{M}$ & $\mathbf{S}$ \\
\hline P05 & 20 & 27 & 17 & 28 \\
\hline P25 & 37 & 40 & 36 & 44 \\
\hline P50 & 51 & 49 & 52 & 54 \\
P75 & 70 & 61 & 75 & 67 \\
P95 & 106 & 80 & 125 & 88 \\
MW & 55 & 51 & 59 & 56 \\
\hline SD & 12 & 19 & 10 & 18 \\
\hline Max & 175 & 240 & 264 & 272 \\
\hline MW Mittelwert, SD Standardabweichung, P05 bis P95 Perzentilwerte & \\
\hline
\end{tabular}

Tab. 5 Gesamthandkräfte beim Befahren einer Rampe und Überwinden einer Stufe mit dem Rollstuhl und einem Dummygewicht von $100 \mathrm{~kg}$

\begin{tabular}{|c|c|c|c|c|c|c|}
\hline \multirow{2}{*}{$\begin{array}{l}\text { Gesamtkraft } \\
\text { an Kraft- } \\
\text { griffen (N) }\end{array}$} & \multicolumn{2}{|l|}{ Rampe } & \multicolumn{4}{|l|}{ Stufe } \\
\hline & Abwärts & Aufwärts & $\begin{array}{l}\text { Abwärts, } \\
\text { Schieben }\end{array}$ & $\begin{array}{l}\text { Abwärts, } \\
\text { Ziehen }\end{array}$ & $\begin{array}{l}\text { Aufwärts, } \\
\text { Schieben }\end{array}$ & $\begin{array}{l}\text { Aufwärts, } \\
\text { Ziehen }\end{array}$ \\
\hline P05 & 33 & 88 & 42 & 53 & 63 & 24 \\
\hline P25 & 44 & 108 & 70 & 126 & 100 & 88 \\
\hline P50 & 54 & 129 & 98 & 169 & 170 & 172 \\
\hline P75 & 68 & 157 & 153 & 214 & 288 & 316 \\
\hline P95 & 102 & 193 & 320 & 336 & 431 & 561 \\
\hline MW & 59 & 133 & 128 & 174 & 203 & 221 \\
\hline SD & 22 & 33 & 86 & 79 & 121 & 184 \\
\hline Max & 189 & 251 & 405 & 361 & 504 & 856 \\
\hline
\end{tabular}

MW Mittelwert, SD Standardabweichung, $P 05$ bis $P 95$ Perzentilwerte

für CUELA-Messwerte (WIDAAN) erzeugt. Die Unterschiede zwischen der Bewegungsart (Ziehen, Schieben, Manövrieren) und den Gewichten (leer, $80 \mathrm{~kg}, 100 \mathrm{~kg}$ ) wurden mit einem t-Test (2-seitig, Signifikanzniveau $p=0,05$ )

\section{Ergebnisse}

\section{Allgemein}

Die Schiebe- und Ziehvorgänge unterschieden sich hinsichtlich der Ausführungsdauer; für das Ziehen des Bettes über den Flur wurde im Mittel etwas mehr Zeit aufgewendet als für das Schieben (-Tab. 2).

Es zeigt sich auch, dass die Unterschiede bezüglich des Geschlechts nur marginal sind. Aufgrund der individuellen Höhenanpassung der Kraftgriffe waren Körperhöhenunterschiede in diesem Versuchsdesign ebenfalls ohne Bedeutung. Insofern wird im Folgenden auf eine getrenntgeschlechtliche Auswertung verzichtet.

\section{Auswertungsparameter}

Im Folgenden werden jeweils die Ergebnisse der Messungen mit den Handkraftgriffen (Gesamtkraft beider Einzelkraftgriffe, Kraftrichtung und resultierendes Kraftmoment zwischen den Kraftgriffen) und dem CUELA-Messsystem dargestellt (Körperhaltungen). Aus diesen Daten werden die Werte für die Kompressionskraft auf L5/S1 und das Moment an L5/S1 berechnet.

\section{Gesamtkraft an Kraftgriffen (Flur und Zimmer)}

Die Werte der Handkräfte (Gesamtkraft beider Kraftgriffe) von jeweils 3 Versuchsdurchgängen aller Probanden und die daraus resultierenden Mittelwerte sind in $\bullet$ Tab. 3 und 4 dargestellt.

Die P50-Werte aller Schiebe- und Ziehversuche (ohne Rampe und Stufe) lagen in einem relativ engen Bereich zwischen 44 und $74 \mathrm{~N}$. Die Werte des P05 lagen zwischen 20 und $37 \mathrm{~N}$, die des P95 zwischen 72 und 161 N. Die Zunahme der Kraftwerte mit steigendem Bett- bzw. Rollstuhlgesamtgewicht ist deutlich $\mathrm{zu}$ erkennen, ebenso die Unterschiede zwischen den Bewegungsarten. Innerhalb der Gewichtsgruppen wurde jeweils beim Manövrieren die größte Spanne zwischen dem P5 und P95 gemessen, die geringste Variation gab es beim Ziehen. Die höchsten Werte des P95 wurden jeweils beim Manövrie- 


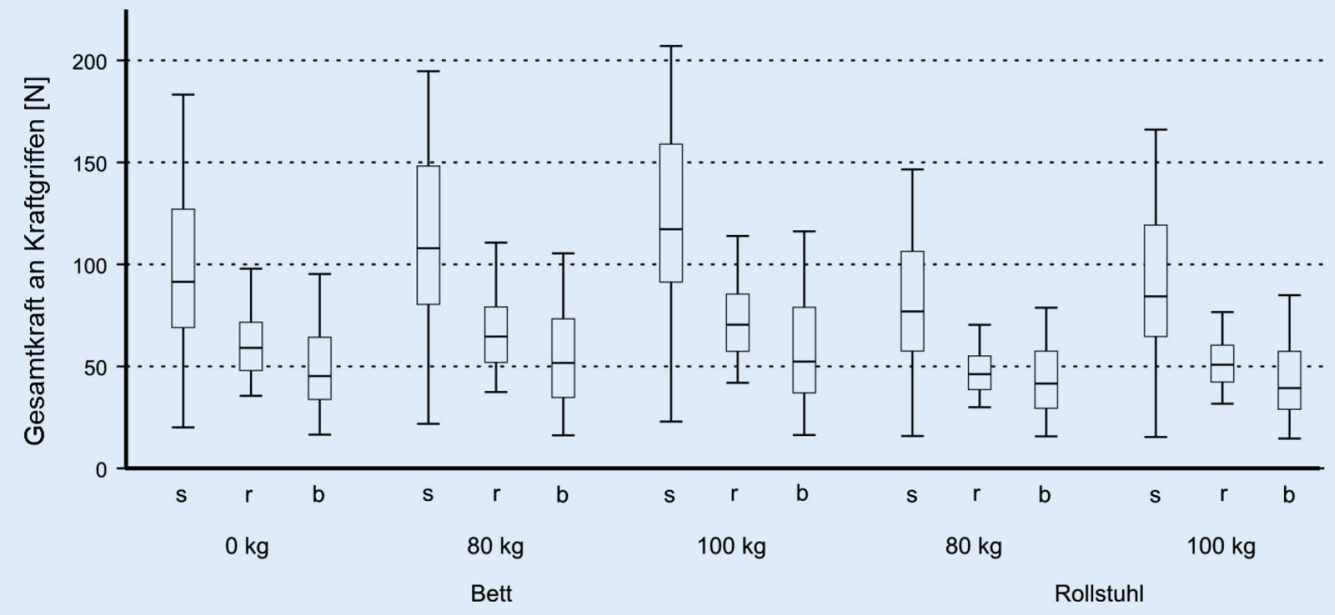

Abb. $4<$ Perzentile der Gesamthandkräfte bei den Start- $(s)$, Roll- $(r)$ und Bremsphasen $(b)$ beim Schieben des Bettes und des Rollstuhls mit verschiedenen Dummygewichten $(0 \mathrm{~kg}, 80 \mathrm{~kg}$ und $100 \mathrm{~kg}$ )

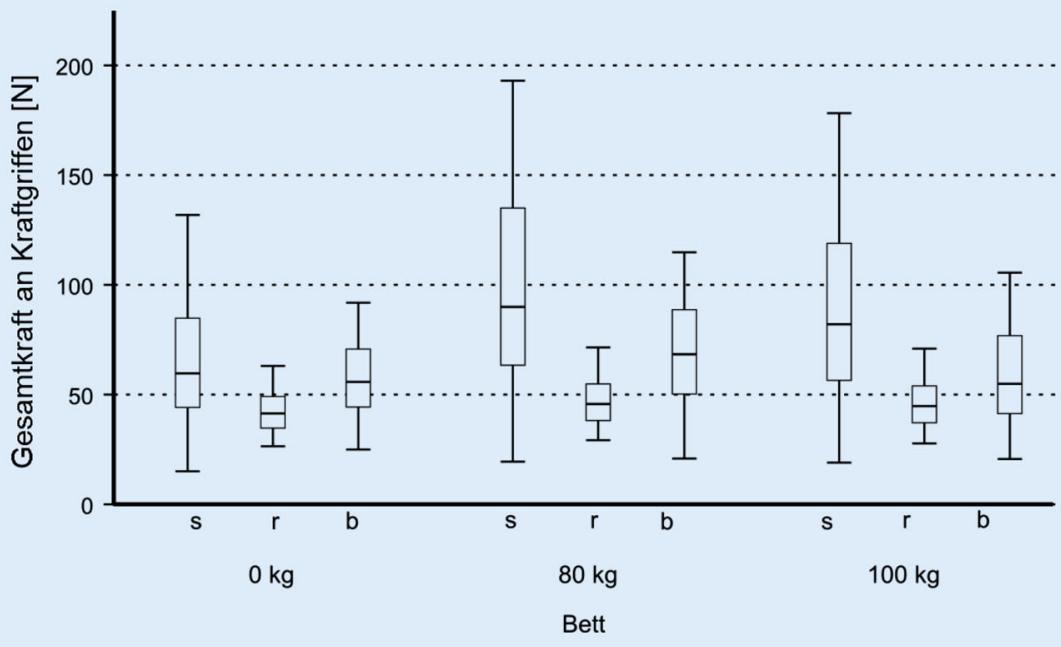

Abb. $5 \Delta$ Boxplots der Gesamthandkräfte bei den Start- $(s)$, Roll- $(r)$ und Bremsphasen $(b)$ beim Ziehen des Bettes mit verschiedenen Dummygewichten $(0 \mathrm{~kg}, 80 \mathrm{~kg}$ und $100 \mathrm{~kg})$

ren erfasst, die geringsten ausschließlich beim Ziehen. Als maximaler Einzelwert wurde $368 \mathrm{~N}$ beim Ziehen des mit einem $80 \mathrm{~kg}$ schweren Dummy beladenen Bettes gemessen.

Nach den Ergebnissen des t-Tests unterscheiden sich die Kraftwerte beim Ziehen, Schieben und Manövrieren hinsichtlich des Gewichts nur zwischen leer und $80 \mathrm{~kg}(p<0,001)$ sowie zwischen leer und $100 \mathrm{~kg}(p<0,001)$. Zwischen 80 und $100 \mathrm{~kg}$ Gewicht wurde kein signifikanter Unterschied festgestellt. Auch für den Rollstuhl wurde kein signifikanter Unterschied zwischen 80 und $100 \mathrm{~kg}$ Dummy berechnet. Hinsichtlich der Bewegungsarten Schieben, Ziehen und Manövrieren bestand zwischen
Manövrieren und Schieben kein signifikanter Unterschied (Bett und Rollstuhl), während der t-Test für die Kombinationen Ziehen-Schieben und ZiehenManövrieren beim Bett hochsignifikant unterschiedliche Werte lieferte (jeweils $p<0,001)$. Dies gilt jeweils für alle Gewichtsbeladungen (leer, 80 und $100 \mathrm{~kg}$ ).

In Anbetracht des variierenden Kraftverlaufs innerhalb der Versuchsteile wurde beim Ziehen und Schieben eine Unterteilung in Startphase, Rollphase und Bremsphase vorgenommen. Da während der Rollphase ebenfalls ein Manövriervorgang in Form einer Kurve vorzunehmen war, wurde dieser Lenkvorgang in die Auswertung nicht mit einbezogen. In - Abb. 4 und 5 sind Boxplot-Verteilungen der charakteristischen Perzentilwerte der gemessenen Gesamthandkraftwerte der einzelnen Start-, Roll- und Bremsphasen dargestellt. Die Perzentilwerte berechnen sich aus den Mittelwerten der Perzentile aller 10 Probanden. Erwartungsgemäß traten die höchsten Werte und der größte Wertebereich beim Schieben und Ziehen während der Startphase auf. Während bei den Schiebeversuchen im Mittel höhere Werte in der Rollphase als in der Bremsphase gemessen wurden, verhält es sich bei den Ziehversuchen umgekehrt. Hier wurden in der Bremsphase höhere Werte als in der Rollphase gemessen. Für die Rollphasen wurden einheitlich vergleichsweise nur geringe Kraftwerte und Standardabweichungen ermittelt. Die Werte des P95 betrugen zwischen $63 \mathrm{~N}$ (Bett, 0 kg, Ziehen, Rollphase) und $207 \mathrm{~N}$ (Bett, $100 \mathrm{~kg}$, Schieben, Startphase).

\section{Kraftgriff Gesamtkraft (Rampe und Stufe)}

Mit dem Rollstuhl wurde mit einem Probanden neben der Schiebe- und Manövrierversuche auch exemplarisch das Überwinden einer Stufe und das Befahren einer Rampe mit jeweils 3 Wiederholungen und schwerem Dummy $(100 \mathrm{~kg})$ durchgeführt. Beim Befahren der Rampe wurden dabei Kraftwerte zwischen $33 \mathrm{~N}$ (P05) und $107 \mathrm{~N}$ (P95) beim Abwärtsschieben und $85 \mathrm{~N}$ (P05) und 193 N (P95) beim Aufwärtsschieben gemessen (• Tab. 5). Beim Überwinden der Stufe betrug das 50. Perzentil bei 
Tab. 6 Momente an Kraftgriffen beim Schieben, Ziehen und Manövrieren des Krankenbettes und des Rollstuhls in den verschiedenen Phasen (Start-, Roll-, und Bremsphase) mit verschiedenen Dummygewichten $(0 \mathrm{~kg}, 80 \mathrm{~kg}, 100 \mathrm{~kg})$

\begin{tabular}{|c|c|c|c|c|c|c|c|c|}
\hline Kraftgriffmoment (Nm) & MW & SD & P5 & P25 & P50 & P75 & P95 & Max \\
\hline Bett_0_Manoever & 13 & 13 & 1 & 3 & 8 & 18 & 39 & 103 \\
\hline Bett_0_Schieben_Roll & 4 & 3 & 0 & 1 & 3 & 5 & 11 & 54 \\
\hline Bett_0_Schieben_Brems & 3 & 2 & 0 & 1 & 2 & 4 & 7 & 16 \\
\hline Bett_0_Schieben_Start & 3 & 2 & 0 & 1 & 2 & 4 & 7 & 18 \\
\hline Bett_0_Ziehen_Roll & 4 & 3 & 1 & 2 & 3 & 6 & 10 & 33 \\
\hline Bett_0_Ziehen_Brems & 3 & 2 & 0 & 1 & 2 & 4 & 7 & 21 \\
\hline Bett_0_Ziehen_Start & 4 & 3 & 0 & 1 & 3 & 5 & 9 & 31 \\
\hline Bett_80_Manoever & 12 & 11 & 1 & 3 & 8 & 16 & 35 & 109 \\
\hline Bett_80_Schieben_Roll & 4 & 3 & 0 & 1 & 3 & 5 & 10 & 38 \\
\hline Bett_80_Schieben_Brems & 2 & 2 & 0 & 1 & 2 & 4 & 7 & 27 \\
\hline Bett_80_Schieben_Start & 3 & 3 & 0 & 1 & 3 & 5 & 8 & 20 \\
\hline Bett_80_Ziehen_Roll & 3 & 3 & 0 & 1 & 3 & 4 & 8 & 35 \\
\hline Bett_80_Ziehen_Brems & 2 & 2 & 0 & 1 & 2 & 3 & 6 & 22 \\
\hline Bett_80_Ziehen_Start & 2 & 2 & 0 & 1 & 2 & 3 & 6 & 19 \\
\hline Bett_100_Manoever & 12 & 13 & 1 & 3 & 8 & 17 & 39 & 111 \\
\hline Bett_100_Schieben_Roll & 4 & 3 & 0 & 1 & 3 & 5 & 10 & 47 \\
\hline Bett_100_Schieben_Brems & 2 & 2 & 0 & 1 & 2 & 4 & 7 & 22 \\
\hline Bett_100_Schieben_Start & 3 & 2 & 0 & 1 & 2 & 4 & 7 & 22 \\
\hline Bett_100_Ziehen_Roll & 4 & 3 & 1 & 1 & 3 & 5 & 9 & 27 \\
\hline Bett_100_Ziehen_Brems & 2 & 2 & 0 & 1 & 2 & 3 & 6 & 29 \\
\hline Bett_100_Ziehen_Start & 3 & 2 & 0 & 1 & 2 & 4 & 8 & 24 \\
\hline Rollstuhl_80_Manoever & 4 & 4 & 1 & 1 & 3 & 5 & 11 & 32 \\
\hline Rollstuhl_80_Schieben_Roll & 2 & 2 & 0 & 1 & 2 & 3 & 6 & 21 \\
\hline Rollstuhl_80_Schieben_Brems & 2 & 1 & 0 & 1 & 1 & 2 & 4 & 12 \\
\hline Rollstuhl_80_Schieben_Start & 2 & 1 & 0 & 1 & 2 & 3 & 5 & 13 \\
\hline Rollstuhl_100_Manoever & 4 & 4 & 0 & 1 & 3 & 6 & 14 & 43 \\
\hline Rollstuhl_100_Schieben_Roll & 2 & 2 & 0 & 1 & 2 & 3 & 6 & 24 \\
\hline Rollstuhl_100_Schieben_Brems & 2 & 2 & 0 & 1 & 1 & 3 & 5 & 14 \\
\hline Rollstuhl_100_Schieben_Start & 2 & 2 & 0 & 1 & 2 & 3 & 5 & 32 \\
\hline
\end{tabular}

Tab. 7 Momente an Kraftgriffen beim Bewegen des Rollstuhls über eine Rampe bzw. Stufe mit $100 \mathrm{~kg}$ Dummygewicht

\begin{tabular}{|l|lllllllll}
\hline Kraftgriffmoment (Nm) & MW & SD & P05 & P25 & P50 & P75 & P95 & Max \\
\hline Rampe abwärts & 3 & 2 & 0 & 1 & 2 & 4 & 7 & 32 \\
Rampe aufwärts & 4 & 3 & 1 & 2 & 4 & 6 & 10 & 24 \\
\hline Stufe abwärts rückwärts & 3 & 3 & 1 & 1 & 2 & 4 & 11 & 14 \\
Stufe abwärts vorwärts & 5 & 4 & 1 & 2 & 4 & 7 & 11 & 16 \\
Stufe aufwärts rückwärts & 5 & 8 & 0 & 1 & 2 & 5 & 27 & 39 \\
Stufe aufwärts vorwärts & 5 & 4 & 1 & 2 & 4 & 7 & 13 & 25
\end{tabular}

MW Mittelwert, SD Standardabweichung, Max Maximum, P05 bis P95 Perzentilwerte allen Kombinationen mit Ausnahme des Abwärtsschiebens $(98 \mathrm{~N})$ etwa $170 \mathrm{~N}$, während das 95. Perzentil und die Maximalwerte deutlich unterschiedlicher ausfielen. Maximal wurden $856 \mathrm{~N}$ erreicht (Stufe, aufwärts, Ziehen).

\section{Kraftrichtung}

Neben der Körperhaltung und der über die Hände eingeleiteten Kräfte ist eine weitere notwendige Komponente zur Abschätzung der körperlichen Belastung die Kraftrichtung. Die mittlere Kraftrichtung der einzelnen Phasen aller Kombinationen ist in Abb. 6, 7 und 8 dargestellt. Die blauen Pfeile markieren die Kraftrichtungen der einzelnen Beladungsgewichte $(0 \mathrm{~kg}, 80 \mathrm{~kg}, 100 \mathrm{~kg})$. Die Länge der Pfeile stellt den Mittelwert der Gesamtkraft der jeweiligen Phase dar.

Die mittlere Kraftrichtung beim Manövrieren ist für eine Belastungseinschätzung nur bedingt aussagekräftig, da eine Einteilung in Roll-, Schub-, und Zugphasen kaum möglich ist und sowohl Schiebe- als auch Ziehvorgänge in ähnlichem Maße und auch gleichzeitig über die beiden Hände vorkommen können. Die resultierende mittlere Kraftrichtung ist hier eine geringe, nahezu senkrecht nach unten wirkende Kraft.

\section{Kraftmomente zwischen den Kraftgriffen}

Die resultierenden Momente zwischen den Kraftgriffen erreichten beim Manövrieren des Bettes erwartungsgemäß die höchsten Werte (-Tab. 6). Sie lagen im Mittel bei $12 \mathrm{Nm}$ (80 und $100 \mathrm{~kg}$ Dummy) bzw. $13 \mathrm{Nm}$ (leeres Bett) und beim Manövrieren des Rollstuhls bei $4 \mathrm{Nm}$ (80 und $100 \mathrm{~kg}$ Dummy). Im 95. Perzentil wurden beim Schieben und Ziehen über eine gerade Strecke Werte zwischen 4 und $11 \mathrm{Nm}$ erreicht. Maximal wurden für das Manövrieren des Bettes 103 bis $111 \mathrm{Nm}$ berechnet. Beim leichteren und einfacher zu manövrierenden Rollstuhl lagen die Maximalwerte bei $32 \mathrm{Nm}$ (80 kg Dummy) und $43 \mathrm{Nm}$ (100 kg Dummy).

Für das Bewegen eines Rollstuhls über eine Rampe oder Stufe wurden im Mittel ähnliche Werte wie beim Schieben oder Ziehen über eine gerade Strecke berechnet (- Tab. 7). Asymmetrische Schiebeoder Ziehvorgänge, die hohe Momente 


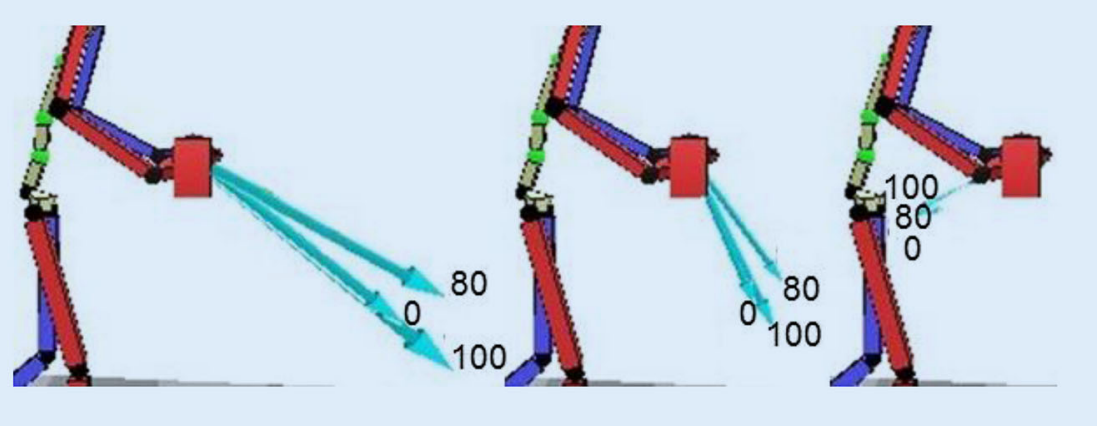

Abb. 6 ॥ Mittlere Kraftrichtungen während Start- (links), Roll- (Mitte) und Bremsphase (rechts) beim Schieben des Bettes mit unterschiedlichen Dummygewichten $(0 \mathrm{~kg}, 80 \mathrm{~kg}, 100 \mathrm{~kg})$. Blaue Pfeile Kraftrichtungen der einzelnen Beladungsgewichte $(0 \mathrm{~kg}, 80 \mathrm{~kg}, 100 \mathrm{~kg})$. Länge der Pfeile Mittelwert der Gesamtkraft der jeweiligen Phase. (Mit freundl. Genehmigung des Verlages Bussert \& Stadeler, [2])

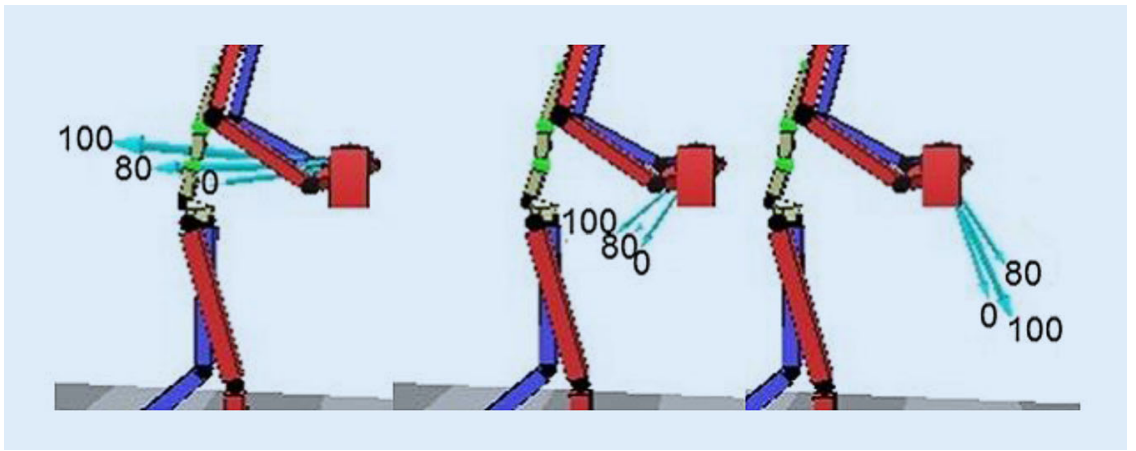

Abb. 7 ॥ Mittlere Kraftrichtungen während Start- (links), Roll- (Mitte) und Bremsphase (rechts) beim Ziehen des Bettes mit unterschiedlichen Dummygewichten $(0 \mathrm{~kg}, 80 \mathrm{~kg}, 100 \mathrm{~kg})$. Blaue Pfeile Kraftrichtungen der einzelnen Beladungsgewichte $(0 \mathrm{~kg}, 80 \mathrm{~kg}, 100 \mathrm{~kg})$. Länge der Pfeile Mittelwert der Gesamtkraft der jeweiligen Phase. (Mit freundl. Genehmigung des Verlages Bussert \& Stadeler, [2])

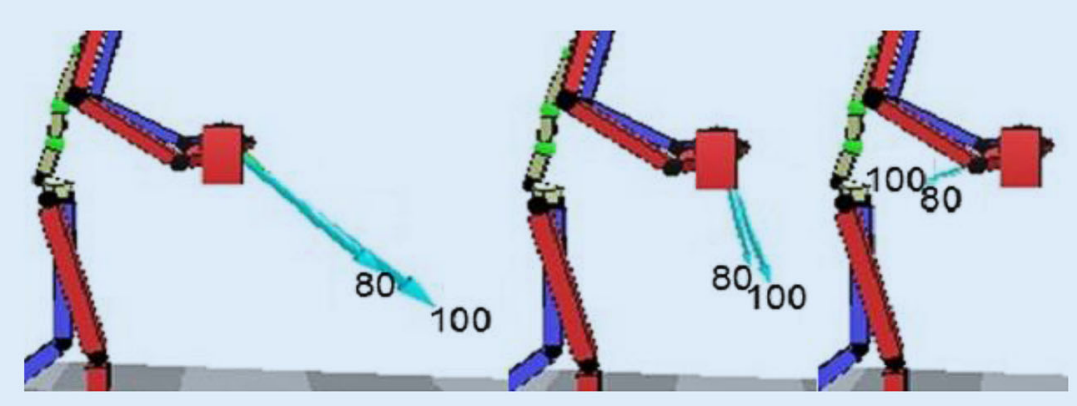

Abb. 8 \& Mittlere Kraftrichtung während Start- (links), Roll- (Mitte) und Bremsphase (rechts) beim Schieben des Rollstuhls mit unterschiedlichen Dummygewichten $(80 \mathrm{~kg}, 100 \mathrm{~kg})$. Blaue Pfeile Kraftrichtungen der einzelnen Beladungsgewichte $(80 \mathrm{~kg}, 100 \mathrm{~kg})$. Länge der Pfeile Mittelwert der Gesamtkraft der jeweiligen Phase

zur Folge haben könnten, wurden dabei nicht durchgeführt.

\section{Körperhaltungen}

Während der verschiedenen Versuche wurden in den Start-, Roll- und Bremsphasen die Häufigkeitsverteilungen der Rückenkrümmung und Rumpfneigung
Im Gegensatz dazu waren die Unterschiede zwischen den unterschiedlichen Phasen beim Rumpfneigungswinkel deutlicher zu erkennen. Bei Schiebevorgängen kommen „leicht überstreckte Haltungen“, d. h. Körperhaltungen mit Rumpfneigungen nach hinten (bis maximal $-5^{\circ}$ ) regelmäßig während der Bremsphase vor, während bei den Ziehversuchen leicht überstreckte Haltungen nur während der Start- und Rollphasen auftraten. Leicht gekrümmte Haltungen mit Rumpfneigungen nach vorne von über $20^{\circ}$ traten nur während der Startphasen beim Schieben des Bettes auf. Für alle anderen Versuchskombinationen wurden Rumpfneigungen im neutralen Bereich verzeichnet.

Bei den Manövriervorgängen wurden innerhalb der Perzentilgrenzen (P5 bis P95) keine negativen Rumpfneigungsund Rückenkrümmungswinkel gemessen. Die Neigung und Krümmung nach vorne ist auch hier abhängig von der zu bewegenden Masse und steigt mit dieser leicht an.

Die Neigungen, Krümmungen und Torsionen des Oberkörpers zur rechten und linken Seite waren auch bei den Manövriervorgängen, bei denen es zu erwarten gewesen wäre, nur sehr gering ausgeprägt und lagen beim 5. und 95. Perzentil jeweils deutlich unterhalb von $10^{\circ}$, die laut Delleman [3] die Grenze zwischen neutraler und leicht gekrümmter Haltung bzw. Torsion darstellen.

Die mit dem Körperhaltungsmesssystem CUELA gemessenen Gelenkwinkel und Körperhaltungen gehen zusammen mit den berechneten Momenten aus den Kraftgriffdaten in die Berechnung der Lumbalmomente und Kompressionskräfte an L5/S1 ein.

\section{Kompressionskräfte und Lumbalmomente an L5/S1}

Während für das Schieben und Ziehen des Bettes und des Rollstuhls auf gerader Strecke im 95. Perzentil Werte von bis zu $45 \mathrm{Nm}$ Drehmoment und 1,2 kN Kompressionskraft berechnet wurden (- Abb. 9 und 10), erreichten die P95Werte beim Manövrieren teilweise über $80 \mathrm{Nm}$ und über 1,6 kN Kompressionskraft. Im 95. Perzentil wurden beim Manövrieren des Bettes bei allen Ge- 


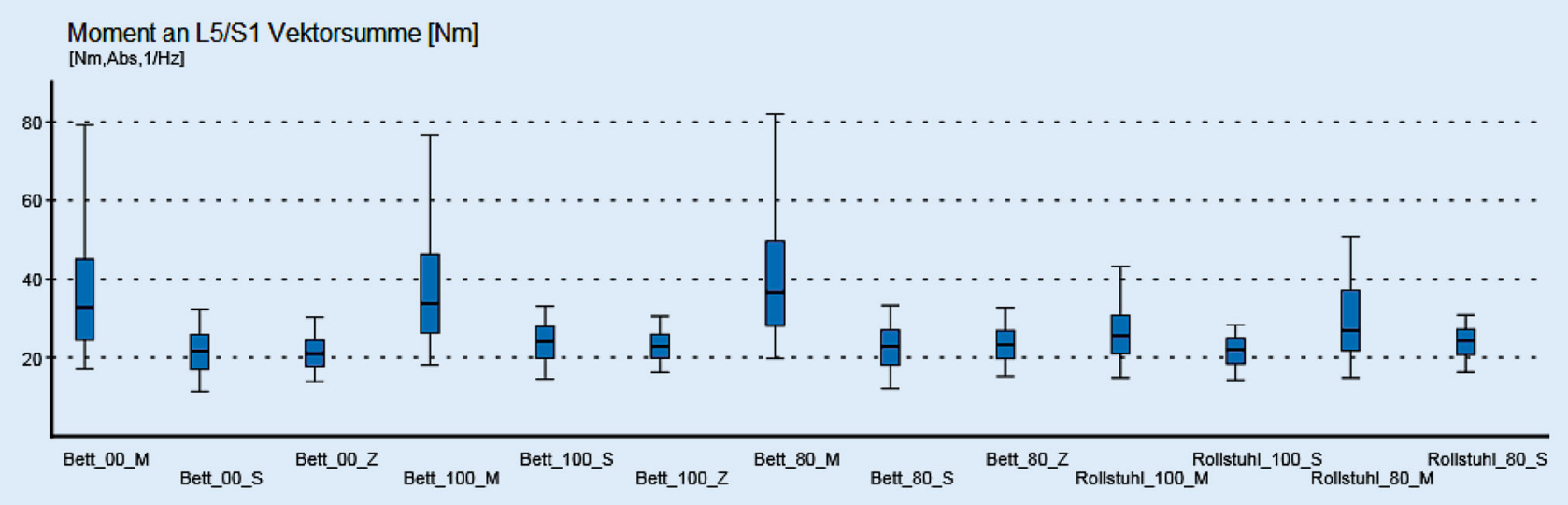

Abb. 9 ム Boxplots der Momente an L5/S1 beim Schieben (S), Ziehen (Z) und Manövrieren (M) des Bettes und des Rollstuhls mit verschiedenen Dummygewichten $(0 \mathrm{~kg}, 80 \mathrm{~kg}$ und $100 \mathrm{~kg}$ )

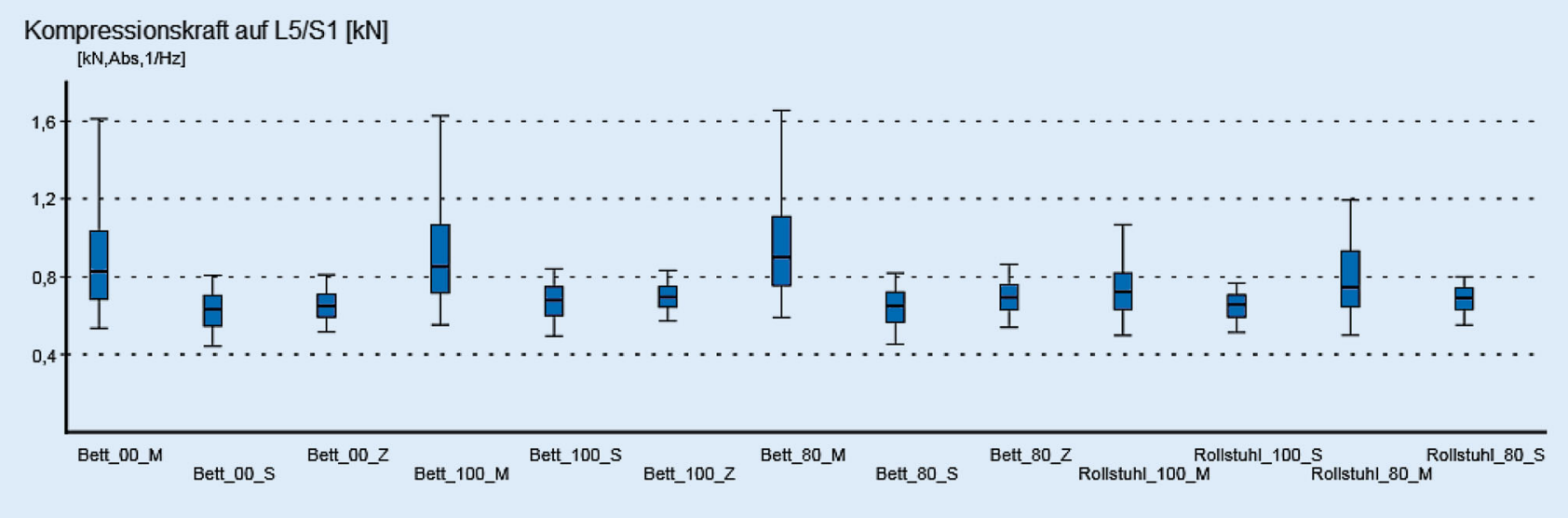

Abb. 10 ॥ Boxplots der Kompressionskraft an L5/S1 beim Schieben (S), Ziehen (Z) und Manövrieren ( $M$ ) des Bettes und des Rollstuhls mit verschiedenen Dummygewichten $(0 \mathrm{~kg}, 80 \mathrm{~kg}$ und $100 \mathrm{~kg})$

wichtsbeladungen etwa $80 \mathrm{Nm}$ erreicht. Die Mediane streuen bei den Versuchen (Bett und Rollstuhl, alle Bewegungsarten) in einem engen Bereich zwischen 22 und $35 \mathrm{Nm}$ Drehmoment und 0,7 und 1,0 kN Kompressionskraft.

Die Drehmomente und Kompressionskräfte an L5/S1 in den verschiedenen Phasen beim Schieben und Ziehen des Bettes (•Tab. 8 und 9) erreichten während der Startphase des zu ziehenden Bettes deutlich höhere Werte als in den übrigen Phasen. Im 95. Perzentil werden hier Werte zwischen 50 und $72 \mathrm{Nm}$ Drehmoment, bzw. 1,2 und 1,6 kN Kompressionskraft erreicht. Selbst im 75. Perzentil wurden bereits Werte von $35 \mathrm{Nm}$ (leeres Bett), $40 \mathrm{Nm}$ (100 kg) und $48 \mathrm{Nm}$ $(80 \mathrm{~kg})$ gemessen. Die Kompressionskräfte erreichten hier Werte von $0,9 \mathrm{kN}$ (leeres Bett), $1,0 \mathrm{kN}$ (80 kg) und 1,2 kN (100 kg).
Die Momente beim Schieben und Ziehen des Rollstuhls hingegen erreichen in allen Phasen Werte von weniger als $40 \mathrm{Nm}$ Drehmoment und bis zu 1,0 kN Kompressionskraftim 95. Perzentil(MW: $22 \pm 4 \mathrm{Nm}$ bis $27 \pm 7 \mathrm{Nm}$ bzw. $1,2 \pm 0,7 \mathrm{kN}$ bis $1,9 \pm 0,8 \mathrm{kN}$; - Tab. 8 und 9), was nach Tichauer [36] als „leichte Arbeit, die von untrainierten Männern und Frauen unabhängig von der Körperstatur durchgeführt werden kann“ bezeichnet wird.

Die exemplarischen Messungen mit einem Probanden (3 Wiederholungen) beim Befahren einer Rampe und Überwinden einer Stufe mit dem Rollstuhl und einem 100 kg schweren Dummy ergaben die höchsten gemessenen Werte für die Lumbalbelastung. Für das Befahren der Rampe wurden durchschnittlich $44 \pm 9 \mathrm{Nm}$ bzw. 1,2 \pm 0,2 kN (abwärts) und $38 \pm 13$ Nm bzw. 1,0 $\pm 0,2 \mathrm{kN}$ Dreh- moment bzw. Kompressionskraft (aufwärts) berechnet; das 95. Perzentil lag bei etwa $60 \mathrm{Nm}$ bzw. 1,5 kN für beide Bewegungsrichtungen. Erheblich höhere Werte von bis zu $290 \mathrm{Nm}$ bzw. 5,8 kN im 95. Perzentil (aufwärts, vorwärts) wurden beim Überwinden einer Stufe erreicht. Das 50. Perzentil für das Überwinden einer Stufe abwärts lag bei $67 \mathrm{Nm}$ Drehmoment (1,6 kN Kompressionskraft, rückwärts) bzw. $48 \mathrm{Nm}$ Drehmoment (1,3 kN Kompressionskraft, vorwärts; • Tab. 10 und 11).

\section{Diskussion}

In verschiedenen Normen, Handlungsanleitungen und Merkblättern werden maximal empfohlene Handkraftwerte beim Ziehen und Schieben angegeben. Die ISO 11228-2 [23] unterscheidet dabei 
Tab. 8 Moment an L5/S1 beim Schieben und Ziehen während der Start-, Roll- und Bremsphasen mit unterschiedlichen Dummygewichten $(0 \mathrm{~kg}, 80 \mathrm{~kg}$ und $100 \mathrm{~kg})$

\begin{tabular}{|c|c|c|c|c|c|c|c|c|}
\hline $\begin{array}{l}\text { Moment an L5/S1 Vektorsumme } \\
(\mathrm{Nm})\end{array}$ & Max & MW & SD & P05 & P25 & P50 & P75 & P95 \\
\hline Bett_0_Schieben_Startphase & 82 & 23 & 8 & 12 & 17 & 23 & 28 & 37 \\
\hline Bett_0_Schieben_Rollphase & 100 & 22 & 7 & 11 & 17 & 22 & 26 & 32 \\
\hline Bett_0_Schieben_Bremsphase & 72 & 23 & 6 & 13 & 18 & 22 & 28 & 33 \\
\hline Bett_0_Ziehen_Startphase & 87 & 30 & 10 & 17 & 22 & 28 & 36 & 50 \\
\hline Bett_0_Ziehen_Rollphase & 63 & 21 & 5 & 14 & 18 & 21 & 24 & 30 \\
\hline Bett_0_Ziehen_Bremsphase & 65 & 21 & 5 & 13 & 17 & 21 & 24 & 30 \\
\hline Bett_80_Schieben_Startphase & 97 & 23 & 9 & 10 & 17 & 22 & 28 & 40 \\
\hline Bett_80_Schieben_Rollphase & 74 & 23 & 7 & 12 & 18 & 23 & 27 & 33 \\
\hline Bett_80_Schieben_Bremsphase & 81 & 28 & 8 & 17 & 22 & 27 & 33 & 43 \\
\hline Bett_80_Ziehen_Startphase & 194 & 40 & 16 & 21 & 27 & 35 & 49 & 73 \\
\hline Bett_80_Ziehen_Rollphase & 66 & 24 & 5 & 15 & 20 & 23 & 27 & 33 \\
\hline Bett_80_Ziehen_Bremsphase & 56 & 23 & 6 & 13 & 19 & 23 & 27 & 34 \\
\hline Bett_100_Schieben_Startphase & 83 & 24 & 8 & 12 & 18 & 23 & 30 & 39 \\
\hline Bett_100_Schieben_Rollphase & 61 & 24 & 6 & 15 & 20 & 24 & 28 & 33 \\
\hline Bett_100_Schieben_Bremsphase & 91 & 26 & 7 & 16 & 21 & 25 & 31 & 38 \\
\hline Bett_100_Ziehen_Startphase & 135 & 34 & 11 & 20 & 25 & 31 & 41 & 56 \\
\hline Bett_100_Ziehen_Rollphase & 69 & 23 & 4 & 16 & 20 & 23 & 26 & 31 \\
\hline Bett_100_Ziehen_Bremsphase & 70 & 23 & 7 & 14 & 19 & 23 & 27 & 35 \\
\hline Rollstuhl_80_Schieben_Startphase & 77 & 25 & 8 & 13 & 19 & 24 & 29 & 39 \\
\hline Rollstuhl_80_Schieben_Rollphase & 50 & 24 & 5 & 16 & 21 & 24 & 27 & 31 \\
\hline Rollstuhl_80_Schieben_Bremsphase & 90 & 27 & 7 & 18 & 22 & 27 & 32 & 38 \\
\hline Rollstuhl_100_Schieben_Startphase & 71 & 23 & 8 & 11 & 17 & 22 & 27 & 38 \\
\hline Rollstuhl_100_Schieben_Rollphase & 55 & 22 & 4 & 14 & 18 & 22 & 25 & 28 \\
\hline Rollstuhl_100_Schieben_Bremsphase & 75 & 25 & 6 & 16 & 20 & 24 & 29 & 36 \\
\hline
\end{tabular}

die „initial force“ von der "sustained force", die mit den in dieser Untersuchung als Start- bzw. Brems- und Rollphase bezeichneten Zieh- und Schiebetätigkeiten verglichen werden können. In der Norm werden die jeweils zurückgelegte Distanz und die Frequenz der Schiebeund Ziehtätigkeiten berücksichtigt. Die maximalen Kraftwerte für Frauen bei Distanzen von 2 bis $30 \mathrm{~m}$ und Häufigkeiten von Schiebe-/Ziehtätigkeiten zwischen $1 / 5$ min und $1 / 8 \mathrm{~h}$ werden hier zwischen $60 \mathrm{~N}$ (Schieben, Rollphase, $30 \mathrm{~m}, 1 / 5 \mathrm{~min}$ ) und $230 \mathrm{~N}$ (Ziehen, Startphase, $2 \mathrm{~m}, 1 / 8 \mathrm{~h}$ ) angegeben. Die Werte des 95. Perzentils in der durchgeführten Untersuchung lagen im Bereich dieser Empfehlung bei $64 \mathrm{~N}(\mathrm{P} 50=36 \mathrm{~N})$ bis $221 \mathrm{~N}$ und können diesbezüglich als unkritisch eingestuft werden. Die angegebenen Maximalwerte der ISO 11228-2 für eine Frequenz von nur einem Vorgang je $8 \mathrm{~h}$ wird im Alltag des Pflegepersonals vermutlich deutlich überschritten. Der nächstniedrigere Tabellenwert, dessen Frequenz mit 1/5 min beispielsweise in einem Klinikbetrieb kaum je erreicht werden dürfte, beträgt dagegen $130 \mathrm{~N}$ während der Startphase für das beidhändige Ziehen („maximum acceptable initial force $-90 \%$ of population, female, handle height $89 \mathrm{~cm}, 60 \mathrm{~m}$ pulling distance"). Eine Handkraft von $130 \mathrm{~N}$ in der Startphase wurde während verschiedener Versuchskombinationen mit dem Wert des 75. Perzentils erreicht (Bett mit 80- und 100-kg-Dummy schiebend und Bett mit 80-kg-Dummy ziehend; • Abb. 4 und 5). Da die Tabellenwerte der Norm jedoch mehr auf zyklischere oder höherfrequente Tätigkeiten abzielen und eine Interpolation der Tabellenwerte nicht ohne Weiteres vorgenommen werden kann, ist eine Einordnung und Bewertung der Höhe der auftretenden Kräfte beim Ziehen und Schieben von Kran- kenbetten und Rollstühlen nur unter Vorbehalt möglich.

Die DIN 33411-5 [6] gibt für die maximal aufzubringenden Kräfte Werte zwischen 258 und $403 \mathrm{~N}$ an (Frauen, P95, stehend, freie Körperhaltung, beidhändig, waagerechte Griffe). Eine Bewertung der hier durchgeführten Zieh- und Schiebetätigkeiten ist mit diesen Kraftwerten jedoch ebenfalls nicht empfehlenswert, da es sich um maximale statische Aktionskräfte handelt, die im Stand und auch nur kurzzeitig aufgebracht werden können.

Eastman Kodak [8] geben als empfohlene Limits der Horizontalkraft $225 \mathrm{~N}$ für die Startphase, $112 \mathrm{~N}$ für die Rollphase und $360 \mathrm{~N}$ für Notstopps an. Steinberg et al. [34] empfehlen für einen schädigungsfreien Umgang mit Lasten beim Ziehen und Schieben weniger als etwa $15 \%$ der Maximalkraft bei länger andauernden Belastungen aufzuwenden. Dies entspräche einer Kraft von $34 \mathrm{~N}$ (Frauen) bis $45 \mathrm{~N}$ (Männer) für die Rollphase. Diese Werte wurden bei allen Versuchskombinationen bereits ab dem 75. Perzentil überschritten, wobei fraglich ist, ob das Schieben eines Krankenbettes in einem Krankenhaus zu einer länger andauernden Belastung gerechnet werden kann. Nach der Leitmerkmalmethode [33, 34] zur Beurteilung von Schiebe- und Ziehvorgängen wurde ein Punktwert von 18 errechnet, der folgendermaßen definiert ist: „Erhöhte Belastung, körperliche Überbeanspruchung bei vermindert belastbaren Personen möglich. Für den Personenkreis sind Gestaltungsmaßnahmen sinnvoll."

Die geringeren Kraftwerte der Handkräfte beim Ziehen, die in dieser Untersuchung im Vergleich zum Schieben gemessen wurden, können auf unterschiedliche Ganggeschwindigkeiten und Beschleunigungen bei den verschiedenen Tätigkeiten zurückgeführt werden, da für die Versuchsdurchführung keine zeitlichen Limits vorgegeben wurden. Die Fahrstrecke im Flur wurde rückwärts ziehend (im Mittel 0,75 $\mathrm{ms}^{-1}$ ) langsamer durchschritten als vorwärts schiebend (im Mittel $0,88 \mathrm{~ms}^{-1}$; $\bullet$ Tab. 2.). Die Ursache für die nur marginal höheren Kompressionskräfte und Momente an L5/S1 beim Ziehen gegenüber dem Schieben 
Tab. 9 Kompressionskraft auf L5/S1 beim Schieben und Ziehen während der Start-, Roll- und Bremsphasen mit unterschiedlichen Dummygewichten $(0 \mathrm{~kg}, 80 \mathrm{~kg}$ und $100 \mathrm{~kg})$

\begin{tabular}{|c|c|c|c|c|c|c|c|c|}
\hline Kompressionskraft L5/S1 (kN) & Max & MW & SD & P05 & P25 & P50 & P75 & P95 \\
\hline Bett_0_Schieben_Startphase & 1,8 & 0,7 & 0,1 & 0,5 & 0,6 & 0,7 & 0,8 & 0,9 \\
\hline Bett_0_Schieben_Rollphase & 1,7 & 0,6 & 0,1 & 0,4 & 0,5 & 0,6 & 0,7 & 0,8 \\
\hline Bett_0_Schieben_Bremsphase & 1,6 & 0,7 & 0,1 & 0,5 & 0,6 & 0,7 & 0,8 & 0,9 \\
\hline Bett_0_Ziehen_Startphase & 2,0 & 0,8 & 0,2 & 0,6 & 0,7 & 0,8 & 0,9 & 1,2 \\
\hline Bett_0_Ziehen_Rollphase & 1,4 & 0,7 & 0,1 & 0,5 & 0,6 & 0,6 & 0,7 & 0,8 \\
\hline Bett_0_Ziehen_Bremsphase & 1,5 & 0,6 & 0,1 & 0,5 & 0,6 & 0,6 & 0,7 & 0,8 \\
\hline Bett_80_Schieben_Startphase & 2,1 & 0,7 & 0,2 & 0,4 & 0,5 & 0,6 & 0,8 & 1,0 \\
\hline Bett_80_Schieben_Rollphase & 1,4 & 0,6 & 0,1 & 0,5 & 0,6 & 0,6 & 0,7 & 0,8 \\
\hline Bett_80_Schieben_Bremsphase & 1,8 & 0,8 & 0,1 & 0,6 & 0,7 & 0,8 & 0,9 & 1,1 \\
\hline Bett_80_Ziehen_Startphase & 3,7 & 1,0 & 0,3 & 0,7 & 0,8 & 0,9 & 1,2 & 1,6 \\
\hline Bett_80_Ziehen_Rollphase & 1,5 & 0,7 & 0,1 & 0,5 & 0,6 & 0,7 & 0,8 & 0,9 \\
\hline Bett_80_Ziehen_Bremsphase & 1,3 & 0,7 & 0,1 & 0,5 & 0,6 & 0,7 & 0,7 & 0,9 \\
\hline Bett_100_Schieben_Startphase & 1,8 & 0,7 & 0,2 & 0,5 & 0,6 & 0,7 & 0,8 & 1,0 \\
\hline Bett_100_Schieben_Rollphase & 1,4 & 0,7 & 0,1 & 0,5 & 0,6 & 0,7 & 0,7 & 0,8 \\
\hline Bett_100_Schieben_Bremsphase & 1,9 & 0,7 & 0,1 & 0,6 & 0,7 & 0,7 & 0,8 & 0,9 \\
\hline Bett_100_Ziehen_Startphase & 2,9 & 0,9 & 0,2 & 0,7 & 0,8 & 0,9 & 1,0 & 1,3 \\
\hline Bett_100_Ziehen_Rollphase & 1,5 & 0,7 & 0,1 & 0,6 & 0,6 & 0,7 & 0,7 & 0,8 \\
\hline Bett_100_Ziehen_Bremsphase & 1,6 & 0,7 & 0,1 & 0,5 & 0,6 & 0,7 & 0,7 & 0,9 \\
\hline Rollstuhl_80_Schieben_Startphase & 1,7 & 0,7 & 0,1 & 0,5 & 0,6 & 0,7 & 0,8 & 1,0 \\
\hline Rollstuhl_80_Schieben_Rollphase & 1,2 & 0,7 & 0,1 & 0,6 & 0,6 & 0,7 & 0,7 & 0,8 \\
\hline Rollstuhl_80_Schieben_Bremsphase & 1,9 & 0,8 & 0,1 & 0,6 & 0,7 & 0,8 & 0,9 & 1,0 \\
\hline Rollstuhl_100_Schieben_Startphase & 1,6 & 0,7 & 0,1 & 0,4 & 0,6 & 0,6 & 0,8 & 0,9 \\
\hline Rollstuhl_100_Schieben_Rollphase & 1,3 & 0,6 & 0,1 & 0,5 & 0,6 & 0,7 & 0,7 & 0,8 \\
\hline Rollstuhl_100_Schieben_Bremsphase & 1,7 & 0,7 & 0,1 & 0,6 & 0,6 & 0,7 & 0,8 & 0,9 \\
\hline
\end{tabular}

könnte ebenso in den unterschiedlichen Durchführungsgeschwindigkeiten begründet sein. Bei genauerem Betrachten der resultierenden Drehmomente und Kompressionskräfte beim Manövrieren fällt auf, dass mit steigendem manövriertem Gewicht nicht unbedingt ein höheres Moment einhergeht (• Abb. 9 und 10). Eine Erklärung hierfür könnte wiederum die Art der Handhabung sein. Geringere Gewichte werden möglicherweise schneller und mit höheren asymmetrischen Hebelbewegungen der Arme bewegt bzw. manövriert, was zu höheren mechanischen Belastungen im LWS-Bereich führen kann. Grundsätzlich können die im Vergleich zum Ziehen und Schieben relativ hohen Kräfte und Momente beim Manövrieren auf mehrere Faktoren zurückgeführt werden. Es finden häufige Wechsel von Ziehund Schiebevorgängen und damit entsprechend viele Startphasen mit hohen Kraftaufwendungen statt. Neben höhe- ren Kraftaufwendungen bedingt durch die Überwindung des Anfangswiderstandes müssen oftmals auch zusätzliche Widerstände durch quer zur Fahrtrichtung stehende Laufrollen überwunden werden. Asymmetrische Kraftaufwendungen über beide Hände bei den Richtungswechseln verursachen ein relativ hohes Drehmoment zwischen den Händen und in der Lumbalregion L5/S1, was sich auf die Kompressionskraft auswirken kann.

Hoozemans et al. [21] geben an, dass der Zusammenhang von Ziehen und Schieben und Beschwerden im LWS-Bereich zum größten Teil auf gebeugte und verdrehte Haltungen zurückzuführen sei. Während gebeugte Haltungen bei an die Körperhöhe angepassten Griffpositionen weitestgehend vermieden wurden, traten verdrehte oder tordierte Haltungen nur in geringem Maße beim Manövrieren des Bettes auf. Für diese Tätigkeit wurden dennoch, neben dem
Ziehen rückwärts in der Startphase (nach hinten geneigte/überstreckte Körperhaltung), die höchsten Lumbalmomente und Kompressionskräfte berechnet. Die Ergebnisse dieser Studie zeigen somit, dass hohe Belastungen im LWS-Bereich nicht unbedingt an der Körperhaltung allein erkennbar sein müssen oder können. Hohe diagonal wirkende Kräfte und Momente sind $u$. U. nur durch Nutzung anderer Messmethoden, wie z.B. Kraftmessgriffe, erkennbar und für eine über eine Abschätzung hinausgehende detaillierte Berechnung von Momenten und Kompressionskräften an L5/S1 müsste ein entsprechend komplexeres Muskelmodell angewendet werden.

Lee et al. [31, 32] gaben an, dass das Rückwärtsziehen den Lumbalbereich L5/S1 grundsätzlich höher belaste als das Vorwärtsschieben. Diese Ergebnisse werden in dieser Untersuchung mit geringen Unterschieden von bis zu 0,1 kN Kompressionskraft (• Abb. 10) grundsätzlich bestätigt. Die Unterschiede sind dabei hauptsächlich auf die resultierenden Kompressionskräfte und Drehmomente während der Startphase (『Tab.9) zurückzuführen.

Die Drehmomente und Kompressionskräfte an L5/S1 in den verschiedenen Phasen beim Schieben und Ziehen des Bettes (๑Tab. 8 und 9) erreichten während der Startphase des zu ziehenden Bettes deutlich höhere Werte als in den übrigen Phasen. Dies ist plausibel mit ungünstigeren Körperhaltungen zu erklären. Im 95. Perzentil werden hier Werte zwischen 50 und $72 \mathrm{Nm}$ Drehmoment, bzw. 1,2 und 1,6 kN Kompressionskraft erreicht. Selbst im 75. Perzentil wurden bereits Werte von $35 \mathrm{Nm}$ (leeres Bett), $40 \mathrm{Nm}(100 \mathrm{~kg})$ und $48 \mathrm{Nm}(80 \mathrm{~kg})$ gemessen (die sich teilweise schon im mittleren Bereich nach der Einteilung von Tichauer befinden [36]). Die Dortmunder Richtwerte [24] wurden allerdings nur beim Ziehen des Bettes (mit 80 und $100 \mathrm{~kg}$ Dummygewicht) in der Startphase und beim Überwinden einer Stufe (nach oben) bei einzelnen Maximalwerten erreicht bzw. überschritten. Für die Werte des 95. Perzentils wurden diese nur bei der Überwindung von Stufen überschritten. 
Tab. 10 Moment an L5/S1 beim Befahren einer Rampe und Überwinden einer Stufe mit Rollstuhl und 100-kg-Dummy

\begin{tabular}{|c|c|c|c|c|c|c|c|c|}
\hline $\begin{array}{l}\text { Moment an L5/S1 Vektor- } \\
\text { summe (Nm) }\end{array}$ & Max & MW & SD & P05 & P25 & P50 & P75 & P95 \\
\hline Rampe abwärts & 95 & 44 & 9 & 31 & 38 & 43 & 48 & 60 \\
\hline Rampe aufwärts & 97 & 38 & 13 & 20 & 28 & 36 & 44 & 62 \\
\hline Stufe abwärts rückwärts & 134 & 63 & 29 & 22 & 39 & 67 & 81 & 119 \\
\hline Stufe abwärts vorwärts & 258 & 60 & 41 & 14 & 31 & 48 & 80 & 144 \\
\hline Stufe aufwärts rückwärts & 366 & 122 & 77 & 49 & 64 & 85 & 163 & 277 \\
\hline Stufe aufwärts vorwärts & 453 & 144 & 85 & 33 & 70 & 111 & 212 & 290 \\
\hline
\end{tabular}

Tab. 11 Kompressionskraft an L5/S1 beim Befahren einer Rampe und Überwinden einer Stufe mit Rollstuhl und 100-kg-Dummy

\begin{tabular}{|c|c|c|c|c|c|c|c|c|}
\hline $\begin{array}{l}\text { Kompressionskraft auf } \\
\text { L5/S1 (kN) }\end{array}$ & Max & MW & SD & P05 & P25 & P50 & P75 & P95 \\
\hline Rampe abwärts & 2,2 & 1,2 & 0,2 & 0,9 & 1,1 & 1,2 & 1,3 & 1,5 \\
\hline Rampe aufwärts & 1,8 & 1,0 & 0,2 & 0,7 & 0,8 & 1,0 & 1,1 & 1,4 \\
\hline Stufe abwärts rückwärts & 3,0 & 1,5 & 0,6 & 0,6 & 1,0 & 1,6 & 1,9 & 2,8 \\
\hline Stufe abwärts vorwärts & 5,1 & 1,5 & 0,8 & 0,6 & 0,9 & 1,3 & 1,8 & 3,1 \\
\hline Stufe aufwärts rückwärts & 7,6 & 2,6 & 1,5 & 1,3 & 1,6 & 1,9 & 3,3 & 5,6 \\
\hline Stufe aufwärts vorwärts & 8,9 & 3,1 & 1,7 & 0,8 & 1,7 & 2,4 & 4,4 & 5,8 \\
\hline
\end{tabular}

\section{Limitationen}

Bei der Interpretation der Ergebnisse der hier durchgeführten Untersuchung sind einige Limitationen zu berücksichtigen. Die Zieh- und Schiebeversuche wurden unter standardisierten Laborbedingungen (z. B. mit weitestgehend idealen Bodenoberflächen) mit lediglich 10 Probanden, die nicht als Krankenpflegefachkräfte ausgebildet waren, bei Handhabung eines spezifischen Bettund Rollstuhltyps durchgeführt. Die Abschätzung der Kompressionskräfte erfolgte mit einer einfachen biomechanischen Modellrechnung. Insbesondere für Manövriertätigkeiten und für die Startphase könnte deshalb die Anwendung komplexerer Muskelmodelle (wie z. B. bei Jäger et al. 26) zu höheren Muskel- und Kompressionskräften führen, da diese zusätzlich asymmetrische Körperhaltungen und Kraftausübungen berücksichtigen. Die situationsbedingt neben der Kompressionskraft auf L5/S1 entstehenden Scherkäfte, die in verschiedenen Raumrichtungen auftreten können, werden in dieser Studie nicht behandelt. Daher erscheint die Nutzung der hier ermittelten Belastungswerte als chanische Modelle empfehlenswert.

\section{Fazit für die Praxis}

- Die Ergebnisse und Empfehlungen früherer Studien zu Muskel-SkelettBelastungen bei Zieh- und Schiebetätigkeiten konnten mit Einschränkungen wegen unterschiedlicher Methoden bestätigt werden.

- Weitere Untersuchungen mit einem größeren Probandenkollektiv sollten jedoch zur Verifizierung der Ergebnisse durchgeführt werden.

- In einer Folgestudie sollte auch der Einfluss von unterschiedlichen Bodenbelägen untersucht werden, der in dieser Untersuchung aus einer idealen, glatten und harten, Oberfläche (Fliesen) bestand.

- Andere Untergründe, wie z. B. Linoleum, mit einer flexibleren Oberfläche könnten möglicherweise zu einer deutlich höheren Kraftaufwendung führen.

\section{Korrespondenzadresse}

\section{Brütting}

Institut für Arbeitsschutz der Deutschen Gesetzlichen Unfallversicherung (IFA) Alte Heerstr. 111, 53757 Sankt Augustin, Deutschland

mark.bruetting@dguv.de

\section{Einhaltung ethischer Richtlinien}

Interessenkonflikt. M. Brütting, I. Hermanns, A. Nienhaus und R. Ellegast geben an, dass kein Interessenkonflikt besteht.

Alle im vorliegenden Manuskript beschriebenen Untersuchungen am Menschen wurden mit Zustimmung der zuständigen Ethik-Kommission, im Einklang mit nationalem Recht sowie gemäß der Deklaration von Helsinki von 1975 (in der aktuellen, überarbeiteten Fassung) durchgeführt. Von allen beteiligten Probanden liegt eine Einverständniserklärung vor.

Open Access. Dieser Artikel wird unter der Creative Commons Namensnennung 4.0 International Lizenz (http://creativecommons.org/licenses/by/4.0/deed. de) veröffentlicht, welche die Nutzung, Vervielfältigung, Bearbeitung, Verbreitung und Wiedergabe in jeglichem Medium und Format erlaubt, sofern Sie den/die ursprünglichen Autor(en) und die Quelle ordnungsgemäßnennen, einen Linkzur Creative Commons Lizenz beifügen und angeben, ob Änderungen vorgenommen wurden.

\section{Literatur}

1. Badura B, Schellschmidt H, Vetter Ch (2006) Fehlzeiten-Report 2004. Gesundheitsmanagement in Krankenhäusern und Pflegeeinrichtungen. Springer, Berlin

2. Brütting $M$, Hermanns I, Nienhaus $A$, Ellegast $R$ (2015) Muskel-Skelett-Belastungen beim Ziehen und Schieben von Krankenbetten und Rollstühlen, In: Dienstbühl I, Stadeler M, Scholle HC (Hrsg) 21. Erfurter Tage, Prävention von Arbeitsbedingten Gesundheitsgefahren und Erkrankungen. Dr. Bussert \& Stadeler, Quedlinburg

3. Delleman N (2000) PrEN 1005-4: Evaluation of working postures in relation to machinery. In: Ergonomics for the new millenium: Proceedings of the Triennial Congress of the International Ergonomics Association and Annual Meeting of the Human Factors and Ergonomics Society. Taylor \& Francis, London

4. De Looze MP, von Greuningen K, Rebel J, Kingma I, Kuijer PPFM (2000) Force direction and physical load in dynamic pushing und pulling. Ergonomics 43:377-3100

5. Deutscher Berufsverband für Pflegeberufe e.V. (DBfK) (2012) Zahlen - Daten - Fakten „Pflege“ Hintergrundinformationen. Berlin

6. DIN Deutsches Institut für Normung e.V (1999) DIN 33411-5, „Körperkräfte des Menschen, Teil 5: Maximale statische Aktionskräfte, Werte". Beuth, Berlin

7. DIN Deutsches Institut für Normung e. V.(2008) DIN EN 1005-4, ,Sicherheit von Maschinen - Menschliche körperliche Leistung - Teil 4: Bewertung von 
Körperhaltungen und Bewegungen bei der Arbeit an Maschinen". Beuth, Berlin

8. Eastman Kodak Company (1986) „Ergonomic design for people at work", Ergonomics Group, Health and Environment Laboratories Bd. 2. Van Nostrand Reinhold, New York

9. Ellegast RP (1998) „Personengebundenes Meßsystem zur automatisierten Erfassung von Wirbelsäulenbelastungen bei beruflichen Tätigkeiten." BIA-Report 5/98. Hauptverband der gewerblichen Berufsgenossenschaften, Sankt Augustin

10. Ellegast RP, Hermanns I, Schiefer Ch (2010) Feldmesssystem CUELA zur Langzeiterfassung und -analyse von Bewegungen an Arbeitsplätzen ZArbeitswiss 64:101-110

11. Estryn-Behar $M$, Kaminski $M$, Peigne $E$, Maillard MF, Pelletier A, Berthier C, Delaporte MF, Paoli MC, Leroux JM (1990) Strenuous working conditions and musculo-skeletal disorders among female hospital workers. Int Arch Occup Environ Health 62:47-57

12. Freitag $S$, Fincke I, Dulon M, Ellegast R, Nienhaus A (2007) Messtechnische Analyse von ungünstigen Körperhaltungen bei Pflegekräften - eine geriatrische Station im Vergleich mit anderen Krankenhausstationen. ErgoMed 5: 130-140

13. Freitag S, Ellegast R, Dulon M, Nienhaus A (2007) Quantitative measurement of stressful trunk postures in nursing professions. Ann Occup Hyg 51(4):385-395

14. Freitag $S$, Fincke-Junod I, Seddouki R, Dulon $M$, Hermanns I, Kersten JF, Larsson TJ, Nienhaus A (2012) Frequent bending - an underestimated burden in nursing professions. Ann Occup Hyg 56(6):697-707

15. Freitag S, Seddouki R, Dulon M, Kersten JF, Larsson TJ, Nienhaus A (2014) The effect of working position on trunk posture and exertion for routine nursing tasks: an experimental study. Ann Occup Hyg 58(3):317-325

16. Gerisch S, Oberlies J (2011) Demografiefeste Personalpolitik in der Krankenhauspflege. Bedarfe und Handlungsstrategien. Leitfaden für die Bildungspraxis, Bd. 40.W. Bertelsmann, Bielefeld

17. Glitsch U, Ottersbach HJ, Ellegast RP, Hermanns I, Feldges W, Schaub K-H, Berg K, Winter G, Sawatzki K, Voß J, Göllner R, Jäger M, Franz G (2004) „Untersuchung der Belastung von Flugbegleiterinnen und Flugbegleitern beim Schieben und Ziehen von Trolleys in Flugzeugen", BIAReport 5/2004. Hauptverband der gewerblichen Berufsgenossenschaften (HVBG), Sankt Augustin

18. Glitsch U, Ottersbach HJ, Ellegast RP, Schaub K-H, Jäger M, Franz G (2007) Physical workload of flight attendants when pushing and pulling trolleys aboard aircraft. Int J Ind Ergon 37:845-854

19. Hecktor K, Jäger M (1994) Beurteilung von Analyseergebnissen und Ableitung von Gestaltungsvorschlägen. In: Laurig W, Hecktor K, Jäger $M$ (Hrsg) Entwicklung eines Expertensystems zur ergonomischen Analyse und Gestaltung von Tätigkeiten des manuellen Lastentransports: ErgonEXPERT. Dokumentation Arbeitswissenschaft, Bd. 36. Otto Schmidt, Köln, S 121-146

20. Hoozemans MJM, van der Beek AJ, Frings-Dresen MHW, van Dijk FJH, van der Woude LHV (1998) Pushing and pulling in relation to musculosceletal disorders: a review of risk factors. Ergonomics 41(6):757-781

21. Hoozemans MJM, van der Beek AJ, Frings-Dresen MHW, van der Woude LHV, van Dijk FJH (2002) Pushing and pulling in association with low back and shoulder complaints. Occup Environ Med 59:696-702
22. Hoozemans MJM, Kuijer PPFM, Kingma I, van Dieen $\mathrm{JH}$, de Vries WHB, van der Woude LHV, van der Beek AJ, Veeger DJ, van der BeekAJ, Frings-Dresen MHW (2004) Mechanical loading of the low back and shoulders during pushing and pulling activities. Ergonomics 47(1):1-18

23. DIN Deutsches Institut für Normung e. V. (2007) ISO 11228-2, „Ergonomie-Manuelle Handhabung - Teil 2:Ziehen und Schieben". Beuth, Berlin

24. JägerM,Luttmann A, GöllnerR(2001)Belastbarkeit der Lendenwirbelsäule beim Handhaben von Lasten - Ableitung der ,Dortmunder Richtwerte' auf Basis der lumbalen Kompressionsfestigkeit. Zentralbl Arbeitsmed 51:354-372

25. Jäger $M$, Theilmeier A, Jordan C, Luttmann A (2005) Dortmunder Lumbalbelastungsstudie 3 - Ermittlung der Belastung der Lendenwirbelsäule bei ausgewählten Pflegetätigkeiten mit Patiententransfer. Teil 2: Belastungskennwerte von sicher gefährdenden Tätigkeiten im Sinne der Berufskrankheit 2108. Shaker, Aachen

26. Jäger M, Sawatzki K, Glitsch U, Ellegast RP, Ottersbach H-J, Schaub K-H, Franz G, Luttmann A (2007) Load on the lumbar spine of flight attendants during pushing and pulling trolleys aboard aircrafts. Int J Ind Ergon 37:863-876

27. Jäger $M$, Jordan $C$, Theilmeier A, Wortmann N, Kuhn S, Nienhaus A, Luttmann A (2013) Lumbarload analysis of manual patient-handling activities for biomechanical overload prevention among healthcare workers. Ann Occup Hyg 57:528-544

28. Jäger M, Jordan C (2016) Dortmunder Lumbalbelastungsstudie 3 - Ermittlung der Belastung der Lendenwirbelsäule bei ausgewählten Pflegetätigkeiten mit Patiententransfer. Teil 5: Tätigkeitss pezifische praktische Handlungsanleitungen für rückengerechtes Bewegen von Patienten. Shaker Aachen

29. Jordan C, Luttmann A, Theilmeier A, Kuhn S, Wortmann N, Jäger M (2011) Characteristic values of the lumbar load of manual patient handling for the application in workers' compensation procedures. JOccup Med Toxicol 6:17

30. Jordan C, Theilmeier A, Luttmann A, Jäger $M$ (2015) Dortmunder Lumbalbelastungsstudie 3 Ermittlung der Belastung der Lendenwirbelsäule bei ausgewählten Pflegetätigkeiten mit Patiententransfer. Teil 4: Biomechanische Bewertung der Belastung der Lendenwirbelsäule von Pflegepersonen beim Bewegen von schwergewichtigen Patienten. Shaker, Aachen

31. Lee KS, Chaffin DB, Herrin GD, Waiker AM (1991) Effect of handle height on lower-back loading in cart pushing and pulling. Appl Ergon 22:117-123

32. Lee KS, Chaffin DB, Parks C (1992) A study of slip potential during cart pushing and pulling. IIE Trans 24(5):139-146

33. Leitmerkmalmethode, Bundesanstalt für Arbeitsschutz und Arbeitsmedizin (BAuA), Nöldnerstr. 40-42, 10317 Berlin, 2008

34. Steinberg U, Caffier G, Liebers F, Behrendt S (2008) Ziehen und Schieben ohne Schaden, 4. Aufl. (Quartbroschüre: Allgemein), Dortmund

35. Tichauer ER (1975) Occupational biomechanics (The anatomical basis of work-place design). Rehabilitation Monograph, Bd. 51. Institute of Rehabilitation Medicine, New York

36. Tichauer ER (1978) The biomechanical basis of ergonomics anatomy applied to the design of work situations. John Wiley \& Sons, New York, S46

\section{Walter K.H. Hoffmann Stimmen der Macht}

Bekenntnisse und Erkenntnisse aus Unternehmen

Zürich: vdf Hochschulverlag AG an der ETH Zürich 2016, 1. Auflage, 304 S., (ISBN: 978-3-7281-3763-0), 38,00 EUR

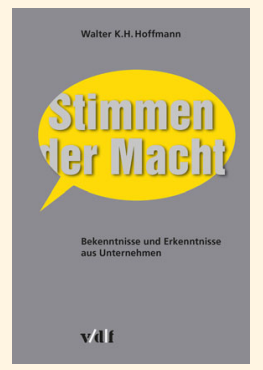

"Stimmen der

Macht" bietet in 35 detaillierten Interviews einen konstruktiv-kritischen Blick auf die Bedeutung der Macht in der heutigen Gesellschaft. Zu den Befragten, die zugunsten größtmöglicher Offenheit im Interview anonym bleiben, gehören Vertreter der Machtelite und Jungunternehmer, aber auch fünf unabhängige Machtbeobachter.

Es werden nationale und internationale Machtnetzwerke und -gruppierungen, wie Banken und Ratingagenturen, facettenreich beleuchtet. Durch den Einblick, der hier aus erster Hand geboten wird, ergibt sich ein differenziertes Bild über die Ideologien und Handlungsweisen aktueller und vergangener Machtstrukturen. So wird auch der Leser zur Selbstreflexion und -analyse ermutigt.

Zudem der Ausblick in die Zukunft: wie sehen die Werte künftiger mächtiger Firmen aus? Stehen bald Eigenständigkeit und Teamgeist statt Geld und Status im Vordergrund?

D.A. Groneberg (Frankfurt am Main) 